\title{
A threshold of transmembrane potential is required for mitochondrial dynamic balance mediated by DRP1 and OMA1
}

\author{
Edith Jones $^{1} \cdot$ Norma Gaytan $^{1} \cdot$ Iraselia Garcia $^{1} \cdot$ Alan Herrera $^{1} \cdot$ Manuel Ramos $^{1} \cdot$ \\ Divya Agarwala $^{1}$ Maahrose Rana ${ }^{1}$ - Wendy Innis-Whitehouse ${ }^{2} \cdot$ Erin Schuenzel $^{1}$. \\ Robert Gilkerson ${ }^{1,3}$ (i)
}

Received: 1 April 2016/Revised: 6 November 2016/Accepted: 14 November 2016/Published online: 17 November 2016 (C) The Author(s) 2016. This article is published with open access at Springerlink.com

\begin{abstract}
As an organellar network, mitochondria dynamically regulate their organization via opposing fusion and fission pathways to maintain bioenergetic homeostasis and contribute to key cellular pathways. This dynamic balance is directly linked to bioenergetic function: loss of transmembrane potential across the inner membrane $\left(\Delta \psi_{\mathrm{m}}\right)$ disrupts mitochondrial fission/fusion balance, causing fragmentation of the network. However, the level of $\Delta \psi_{\mathrm{m}}$ required for mitochondrial dynamic balance, as well as the relative contributions of fission and fusion pathways, have remained unclear. To explore this, mitochondrial morphology and $\Delta \psi_{\mathrm{m}}$ were examined via confocal imaging and tetramethyl rhodamine ester (TMRE) flow cytometry, respectively, in cultured 143B osteosarcoma cells. When normalized to the TMRE value of untreated 143B cells as $100 \%$, both genetic (mtDNA-depleted $\rho^{0}$ ) and pharmacological [carbonyl cyanide m-chlorophenyl hydrazone (CCCP)-treated] cell models below 34\% TMRE fluorescence were unable to maintain
\end{abstract}

E. Jones and N. Gaytan contributed equally.

Electronic supplementary material The online version of this article (doi:10.1007/s00018-016-2421-9) contains supplementary material, which is available to authorized users.

Robert Gilkerson

robert.gilkerson@utrgv.edu

1 Department of Biology, The University of Texas Rio Grande Valley, 1201 West University Drive, Edinburg, TX 78539-2999, USA

2 Department of Biomedical Sciences, The University of Texas Rio Grande Valley, Edinburg, TX 78539-2999, USA

3 Department of Clinical Laboratory Sciences, The University of Texas Rio Grande Valley, 1201 West University Drive, Edinburg, TX 78539-2999, USA mitochondrial interconnection, correlating with loss of fusion-active long OPA1 isoforms (L-OPA1). Mechanistically, this threshold is maintained by mechanistic coordination of DRP1-mediated fission and OPA1-mediated fusion: cells lacking either DRP1 or the OMA1 metalloprotease were insensitive to loss of $\Delta \psi_{\mathrm{m}}$, instead maintaining an obligately fused morphology. Collectively, these findings demonstrate a mitochondrial 'tipping point' threshold mediated by the interaction of $\Delta \psi_{\mathrm{m}}$ with both DRP1 and OMA1; moreover, DRP1 appears to be required for effective OPA1 maintenance and processing, consistent with growing evidence for direct interaction of fission and fusion pathways. These results suggest that $\Delta \psi_{\mathrm{m}}$ below threshold coordinately activates both DRP1-mediated fission and OMA1 cleavage of OPA1, collapsing mitochondrial dynamic balance, with major implications for a range of signaling pathways and cellular life/death events.

Keywords Oxidative phosphorylation - mtDNA .

S-OPA1 · Protonophore $\cdot$ Proteolytic cleavage

$\begin{array}{ll}\text { Abbreviations } \\ \text { CCCP } & \text { Carbonyl cyanide m-chlorophenyl hydrazine } \\ \text { DAPI } & \text { Diaminophenylindole } \\ \text { DMEM } & \text { Dulbecco's Modified Eagle's medium } \\ \text { DMSO } & \text { Dimethylsulfoxide } \\ \text { EtBr } & \text { Ethidium bromide } \\ \text { FBS } & \text { Fetal bovine serum } \\ \text { MEF } & \text { Mouse embryonic fibroblast } \\ \text { mtDNA } & \text { Mitochondrial DNA } \\ \text { OxPhos } & \text { Oxidative phosphorylation } \\ \text { PBS } & \text { Phosphate-buffered saline } \\ \text { TMRE } & \text { Tetramethyl rhodamine ester }\end{array}$


WT Wild type

$\Delta \psi_{\mathrm{m}} \quad$ Mitochondrial transmembrane potential

\section{Introduction}

Mitochondria have emerged as a highly responsive organellar network that dynamically balances between two states: a collection of individual spherical organelles, or an elaborately interconnected reticular network [1,2], thus adapting its organization to directly integrate into crucial cellular processes including metabolism, apoptosis, autophagy, and proliferation [3]. Moreover, mitochondrial dynamics are directly linked with bioenergetic function in an integrated structure/function relationship: loss of transmembrane potential $\left(\Delta \psi_{\mathrm{m}}\right)$, which is critical to oxidative phosphorylation (OxPhos), causes collapse of structural homeostasis, leading to complete fragmentation of the mitochondrial network [4]. Despite this, it has remained unclear what level of bioenergetic function is required for mitochondrial dynamic balance, as well as the contributing mechanisms necessary. Here, our results reveal a 'tipping point' threshold of mitochondrial structure/function.

Mitochondrial dynamics employ an elegant balance of fusion and fission pathways, with each process mediated by a distinct set of interacting factors. Fusion is carried out by optic atrophy-1 (OPA1), a dynamin-related GTPase [5] that mediates fusion of the inner membrane [6,7], while fusion of the outer mitochondrial membrane is accomplished by mitofusins 1 (MFN1) and 2 (MFN2). Fusion of the mitochondrial outer and inner membranes allows individual organelles to exchange components [4] and permits complementation between functional and dysfunctional organelles [8]. The opposing process, fission, is mediated by dynamin-related protein-1 (DRP1) [9], which is recruited to the mitochondrial outer membrane by interacting proteins FIS1 [10, 11] and MFF1 [12] where it forms an oligomeric ring, dividing mitochondria at discrete sites. As these processes are carried out by different sets of interacting factors, inhibition of one pathway causes an increase in the other: inhibition of DRP1-mediated fission causes unopposed mitochondrial fusion [13, 14]. In a unique organellar structure/function relationship, fission/fusion dynamics are increasingly linked to mitochondrial bioenergetics via the transmembrane potential across the inner membrane $\left(\Delta \psi_{\mathrm{m}}\right)$ [15].

Mitochondrial ATP production is accomplished by the five OxPhos complexes in the mitochondrial inner membrane. Complexes I-IV utilize electron transfer to generate the proton-motive $\Delta \psi_{\mathrm{m}}$ that is used by Complex $\mathrm{V}$, the $\mathrm{F}_{1} \mathrm{~F}_{0}$ ATP synthase, to create ATP from ADP and $\mathrm{P}_{\mathrm{i}}$. These complexes are composed of polypeptide subunits encoded on both chromosomal and mitochondrial DNA (mtDNA). Strikingly, cells with partial or complete loss of $\Delta \psi_{\mathrm{m}}$ are unable to maintain an interconnected, reticular mitochondrial morphology: cells depleted of mtDNA ( $\rho^{0}$ cells), as well as cells carrying mtDNA mutations affecting $\Delta \psi_{\mathrm{m}}$, show completely fragmented mitochondrial ultrastructure [16-19], while pharmacological dissipation of $\Delta \psi_{\mathrm{m}}$ via the protonophore CCCP and the ionophore valinomycin also cause fragmentation of the mitochondrial network $[4,20]$. While the mitochondria of mtDNA-depleted $\rho^{0}$ cells show a weak ability to fuse and exchange contents via 'kiss-andrun' events [21], both $\rho^{0}$ and CCCP-treated cells are unable to maintain fission/fusion balance, with their mitochondria existing instead as a fragmented population of organelles $[4,21]$. Despite the importance of $\Delta \psi_{\mathrm{m}}$ to mitochondrial structure/function homeostasis, however, it is unclear i) what level of $\Delta \psi_{\mathrm{m}}$ is required for maintenance of mitochondrial fission/fusion balance, and ii) the relative contribution of the opposing fusion and fission processes to $\Delta \psi_{\mathrm{m}}$-dependent mitochondrial dynamics in the cell.

Fission/fusion dynamics are integral to mitochondrial participation in vital cellular processes: fission is required for stemness [22], mitosis [23, 24], apoptosis [25], and autophagy [26, 27], while fusion is a necessary adaptation to nutrient starvation and increased metabolic demand [3], allowing transmission of $\Delta \psi_{\mathrm{m}}$ along interconnected mitochondria [1]. Moreover, disruption of mitochondrial dynamics and bioenergetics are emerging in prevalent diseases such as heart failure and neurodegenerative disorders $[28,29]$. As such, the interaction of $\Delta \psi_{\mathrm{m}}$ and fission/fusion balance is likely to have major impact as an underlying mechanism of prevalent human disease.

In the classical mitochondrial genetic threshold effect, cells can withstand a high mtDNA mutation load (often up to $80-90 \%$ mutant mtDNA) and maintain full OxPhos function, but when the overall proportion of mutant mtDNA exceeds a critical threshold, mitochondrial bioenergetic function collapses, leading to a variety of systemic and tissue-specific pathologies [30, 31]. Previously, we showed that heteroplasmic cells (carrying both WT and mutant mtDNAs) above $80 \%$ mutation load have completely fragmented mitochondria, while cells below $80 \%$ mutation load have effective fission/fusion balance [17], suggesting that a functional threshold of $\Delta \psi_{\mathrm{m}}$ is required to maintain effective fission/fusion balance. Intriguingly, the OPA1 fusion factor undergoes cleavage and inactivation by the OMA1 metalloprotease in response to dissipation of $\Delta \psi_{\mathrm{m}}$, causing mitochondrial fragmentation [32-34]; however, the level of $\Delta \psi_{\mathrm{m}}$ required and the necessity of coordinating OPA1 cleavage with other fission/fusion factors in balancing mitochondrial dynamics and function remains unclear. 
To explore this, we employed genetic and pharmacological models of decreased $\Delta \psi_{\mathrm{m}}$ to determine the functional and mechanistic requirements for effective mitochondrial fission/fusion dynamic balance in human cells. Cultured cells grown in high-glucose media obtain ATP primarily via glycolysis, allowing the growth and study of cells with genetic or pharmacological defects in bioenergetics [35]. Using TMRE flow cytometry to monitor $\Delta \psi_{\mathrm{m}}$, our data indicate that cells below $34 \%$ of untreated wild-type (WT) TMRE values cannot maintain mitochondrial dynamic balance, with concomitant loss of fusion-active OPA1: this threshold is mediated by DRP1 and OMA1, reflecting a crucial breakpoint of $\Delta \psi_{\mathrm{m}}$ as a determinant of mitochondrial homeostasis, with severe impacts on cell viability and broad relevance to human disease.

\section{Results}

\section{Cells with low $\Delta \psi_{\mathrm{m}}$ lack reticular mitochondrial organization}

Previous studies have found that cells with OxPhos defects have complete fragmentation of the mitochondrial network $[4,16,21]$. To confirm these findings, we examined both genetic and pharmacological cell models of $\Delta \psi_{\mathrm{m}}$ loss in a 143B osteosarcoma cell background. 143B206 $\rho^{0}$ cells have been depleted of all mtDNA through treatment with ethidium bromide (EtBr) [36], resulting in loss of mtDNAencoded polypeptides, $\Delta \psi_{\mathrm{m}}$, and OxPhos function [16, 19]. The protonophore CCCP allows for complete dissipation of the proton gradient across the mitochondrial inner membrane, providing a pharmacological method to eliminate $\Delta \psi_{\mathrm{m}}$. To examine mitochondrial morphology in WT, $\rho^{0}$, and CCCP-treated WT lines, cells were immunolabeled for the TOM20 protein, which is located in the mitochondrial outer membrane, providing imaging of the mitochondrial network in these cells. Mitochondrial localization was confirmed by colocalization with MitoTracker (not shown). WT cells visualized by confocal fluorescence microscopy revealed a balance of both fusion and fission, with highly interconnected, reticular mitochondria existing in the same cell with fragmented individual mitochondria. Conversely, $\rho^{0}$ cells displayed fragmented, somewhat swollen, mitochondria, while WT cells treated with $10 \mu \mathrm{M} \mathrm{CCCP}$ for $1 \mathrm{~h}$ also showed complete fragmentation of the mitochondrial network (Fig. 1a). MtDNA content was verified by PCR from total DNA of indicated cell lines. Amplification of mtDNA nt7130-8113 revealed a strong band in both WT and CCCP-treated WT cells, while $\rho^{0}$ cells showed no mtDNA present (Fig. 1b). Western blot analysis showed that the mtDNA-encoded cytochrome $\mathrm{c}$ oxidase II
(MTCO2) protein was present in WT and CCCP-treated WT cells, but absent in $\rho^{0}$ cells, as expected (Fig. 1c). Consistent with this, immunolabeling microscopy showed strong MTCO2 signal in WT and CCCP-treated WT cells, but not in $\rho^{0}$ cells (not shown). To determine $\Delta \psi_{\mathrm{m}}$, cells were given media containing the $\Delta \psi_{\mathrm{m}}$-dependent dye tetramethyl rhodamine ester (TMRE) and assayed by flow cytometry (representative histograms, Fig. 1d). This assay is a novel adaptation of our previous method [19]. WT cells show a robust average fluorescence of $4172 \pm 172$ arbitrary units (a.u.), while $\rho^{0} \mathrm{~s}$ and CCCP-treated WT cells maintain significantly lower values of $1197 \pm 179$ a.u and $385 \pm 26$ a.u., respectively (Fig. 1e). These values are consistent with those reported for $\rho^{0}$ cells via rhodaminebased evaluation of $\Delta \psi_{\mathrm{m}}$ [21]. Collectively, these findings demonstrate that either genetic or pharmacological decreases in $\Delta \psi_{\mathrm{m}}$ cause complete fragmentation of the mitochondrial network, consistent with previous findings $[4,16]$.

\section{Cells below a threshold of 34\% TMRE fluorescence cannot maintain mitochondrial interconnection}

Intriguingly, $\rho^{0}$ cells maintain a modest, intermediate TMRE signal, statistically greater than that of WT cells treated with $10 \mu \mathrm{M}$ CCCP $[1197 \pm 179$ versus $385 \pm 26$ a.u., respectively (Fig. 1e)], likely due to reversal of the $\mathrm{F}_{1} \mathrm{~F}_{0}$ ATP synthase [37]. Despite this, both have completely fragmented mitochondrial morphology (Fig. 1a). This suggests that a minimum level of $\Delta \psi_{\mathrm{m}}$, greater than that seen in either $\rho^{0}$ or CCCP-treated WT cells, is required for reticular mitochondrial organization. To explore this, WT cells were incubated with a range of concentrations of CCCP for $1 \mathrm{~h}$, and mitochondrial morphology visualized by anti-TOM20 immunolabeling, as above (Fig. 2a). At 0 , 0.1 , and $1 \mu \mathrm{M} \mathrm{CCCP}$, cells displayed full ability to maintain mitochondrial interconnection, as reticular mitochondria were clearly evident. At $10 \mu \mathrm{M} \mathrm{CCCP}$, however, no cells were observed with reticular mitochondria, as all cells had fragmented mitochondria (Fig. 2a), indicating that mitochondrial fission/fusion balance is lost between 1 and $10 \mu \mathrm{M} \mathrm{CCCP.} \mathrm{To} \mathrm{further} \mathrm{probe} \mathrm{this}$ hypothesis, cells were treated with CCCP from 2 to $10 \mu \mathrm{M}$, and TMRE flow cytometry was used to assay $\Delta \psi_{\mathrm{m}}$ as in Fig. 1e. When normalized to untreated WT cells, CCCP at increasing concentrations produced stepwise decreases in TMRE fluorescence, defining a range from untreated (100\%) to $10 \mu \mathrm{M}$ CCCP-treated (9\%). Critically, cells incubated with $4.75 \mu \mathrm{M}$ CCCP showed a normalized TMRE signal of $34 \pm 5 \%$ relative to untreated cells, while cells incubated with $5 \mu \mathrm{M}$ CCCP had TMRE signal of $25 \pm 5 \%$ and $\rho^{0}$ cells $29 \pm 4 \%$ (Fig. 2b). To explore the impact of these stepwise decreases in $\Delta \psi_{\mathrm{m}}$ on 
A
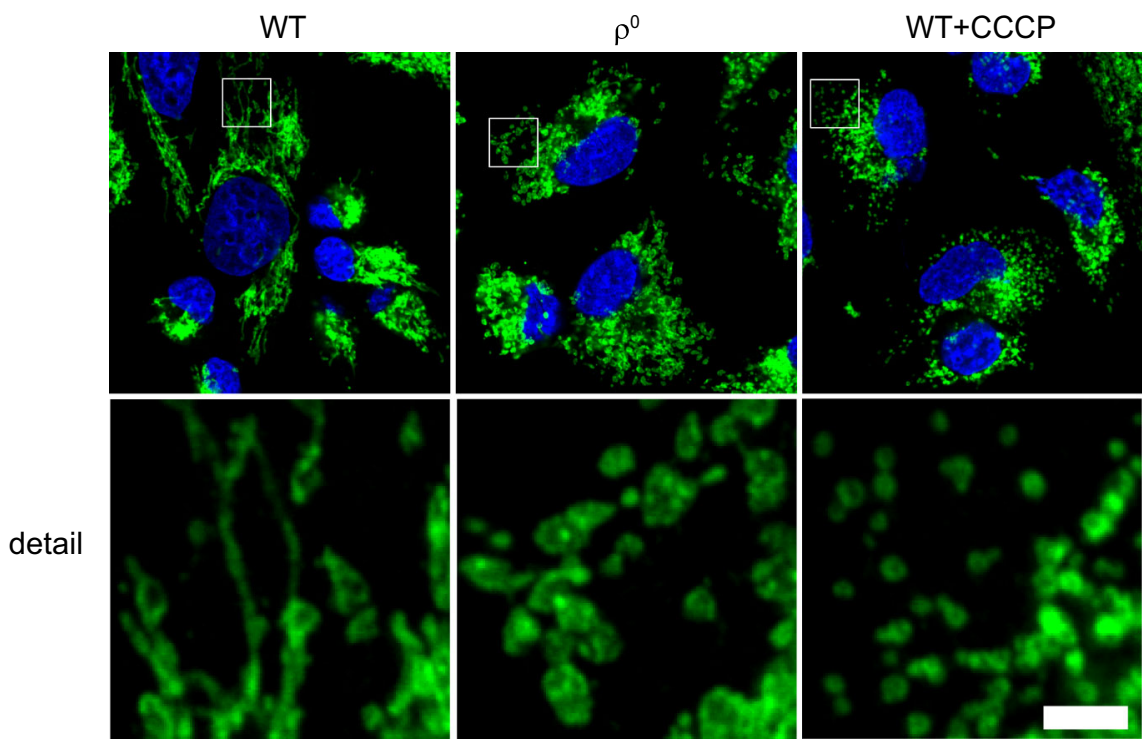

B

B mtDNA nt7130-8113

C

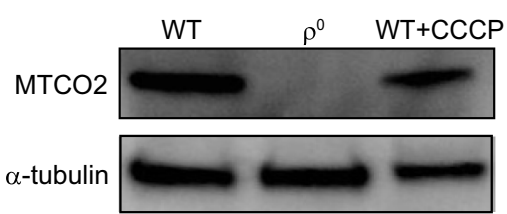

D
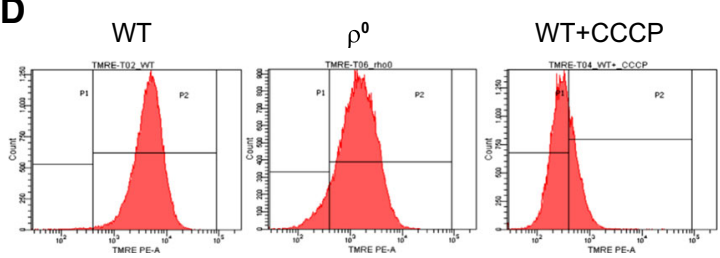

E

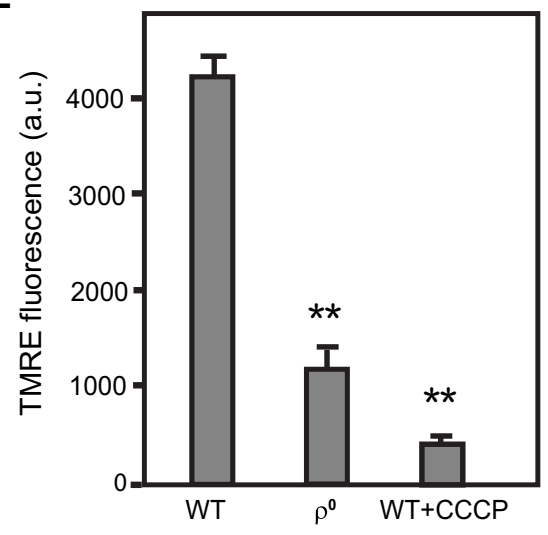

Fig. 1 Mitochondrial morphology and bioenergetics in human 143B osteosarcoma cells. a Cultured 143B cells visualized by confocal fluorescence microscopy. WT, mtDNA-depleted $\rho^{0}$, and CCCPtreated $(10 \mu \mathrm{M}, 1 \mathrm{~h}) \mathrm{WT}$ cells were immunolabeled for mitochondrial TOM20 (green). Nuclei stained with DAPI (blue). Outlined box in Merge (white) is enlarged in Detail panel. Size bar $2 \mu \mathrm{m} . n=3$ experiments. b Polymerase chain reaction of mtDNA. MtDNAspecific primers were used to amplify nt7130-8113 of human mtDNA from $100 \mathrm{ng}$ of total cellular DNA isolated from WT, $\rho^{0}$, and CCCPtreated WT cells. $n=3$ experiments. c Anti-MTCO2 Western blotting of lysates from cultured 143B cell lines. Anti- $\alpha$-tubulin

mitochondrial morphology, cells treated with the indicated concentrations of CCCP were immunolabeled for TOM20. For quantitation, individual cells were scored as having predominantly reticular, fragmented, or intermediate mitochondrial morphologies, as elsewhere [34, 38-40]. When scoring cell profiles, individual cells with more than two regions of either reticular or fragmented mitochondrial organization were scored as intermediate. As expected, untreated cells showed a high proportion of cells with predominantly reticular $(44 \pm 17 \%)$ and intermediate provides loading control. d Representative histograms of WT, $\rho^{0}$, and CCCP-treated WT cells incubated with $100 \mathrm{nM}$ TMRE and assayed via flow cytometry. 50,000 cells assayed for TMRE fluorescence $(X$ axis) in each experiment. Y-axis indicates number of cells at fluorescence values expressed in arbitrary units (a.u.). e Average TMRE fluorescence of WT, $\rho^{0}$, and CCCP-treated WT cells in arbitrary units (a.u.). Values represent average TMRE fluorescence of indicated cell lines in $n>3$ experiments \pm standard error (SE). **Statistical significance, $p<0.01$, Tukey's post hoc test following one-way ANOVA

morphologies $(51 \pm 15 \%)$, with a small $(4 \pm 3 \%)$ proportion of cells with predominantly fragmented mitochondria. On the other hand, cells treated with $10 \mu \mathrm{M}$ CCCP showed 100\% fragmented morphology (Fig. 2c; Table 1), demonstrating the spectrum of mitochondrial morphology and $\Delta \psi_{\mathrm{m}}$ in 143B cells. Strikingly, cells with intermediate $\Delta \psi_{\mathrm{m}}$ levels showed a clear threshold effect: at 0,2 , and $4 \mu \mathrm{M} \mathrm{CCCP},>90 \%$ of cells assayed had either reticular or intermediate morphologies, with less than $15 \%$ of cells having a fragmented morphology at these 
A
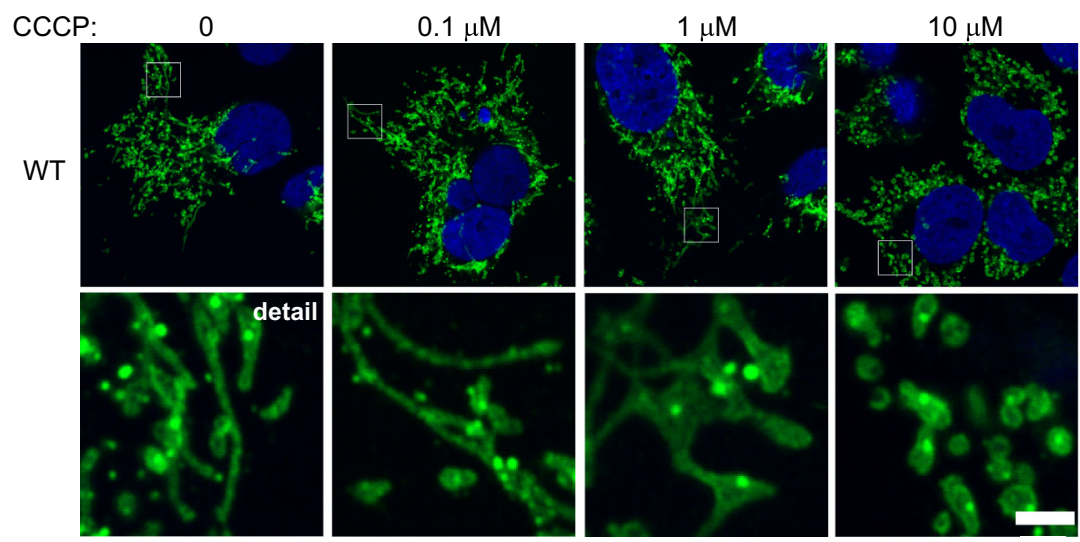

B

D $\mathrm{CCCP}: 4 \mu \mathrm{M}$
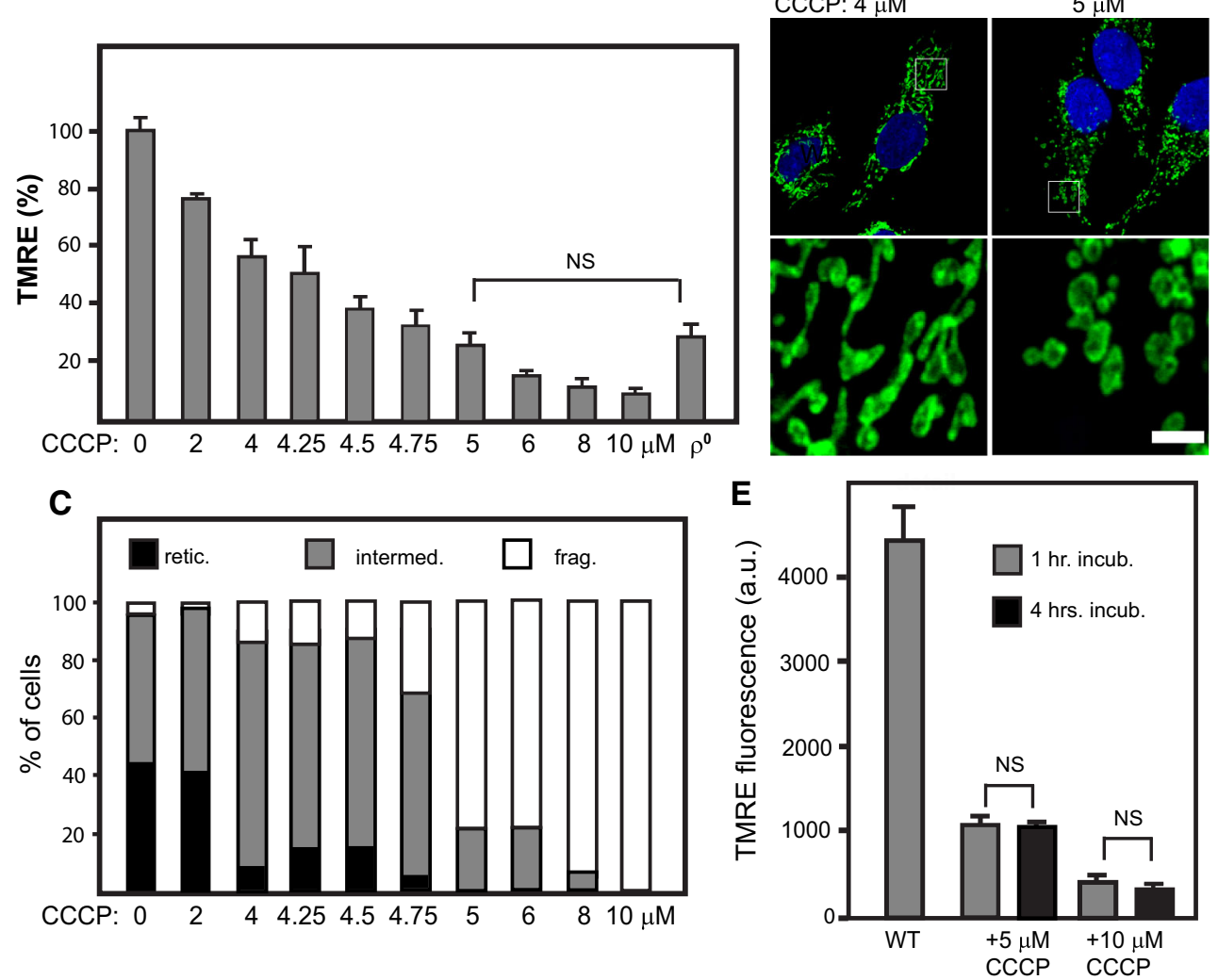

Fig. 2 Mitochondrial morphology and $\Delta \psi_{\mathrm{m}}$ monitored across CCCP titration. a WT cells incubated with $0,0.1,1$, or $10 \mu \mathrm{M} \mathrm{CCCP} \mathrm{for} 1 \mathrm{~h}$ were then fixed and immunolabeled for mitochondrial TOM20 (green), followed by DAPI staining of nuclei (blue). Outlined boxes (white) enlarged in Detail image. Size bar $2 \mu \mathrm{m} . n=3$ experiments. b WT cells were incubated with $0,2,4,4.25,4.5,4.75,5,6,8$, or $10 \mu \mathrm{M}$ CCCP for $1 \mathrm{~h}$, as well as $\rho^{0}$ cells, and incubated with $100 \mathrm{nM}$ TMRE for $20 \mathrm{~min}$. Average TMRE fluorescence given for each condition, expressed as $\%$ of untreated $(0 \mu \mathrm{M}$ CCCP $) \pm$ SE. $n \geq 3$ experiments for each condition, 50,000 cells assayed per experiment. All values analyzed by one-way ANOVA, $p<0.01$. c Quantitation of mitochondrial morphology across CCCP titration. WT cells were incubated with CCCP as in $\mathbf{b}$ for $1 \mathrm{~h}$ and processed for TOM20

concentrations. Conversely, at 5, 6, 8, and $10 \mu \mathrm{M}$, at least $80 \%$ of cells had predominantly fragmented mitochondrial morphology (Fig. 2c; Table 1). A sharp dropoff was immunolabeling, as in a. In three independent experiments, cells were imaged and scored as having predominantly reticular (black), predominantly fragmented (white), or intermediate (gray) mitochondrial morphology. Average \% of cells with indicated morphology shown across CCCP concentrations indicated. For analysis of statistical significance, see Table 1. d WT cells incubated in the presence of 4 or $5 \mu \mathrm{M} \mathrm{CCCP}$ and immunolabeled for TOM20 (green). Size bar $2 \mu \mathrm{m}$. e Cells were incubated in the absence or presence of 5 or $10 \mu \mathrm{M} \mathrm{CCCP}$ for 1 or $4 \mathrm{~h}$ and assayed by TMRE as in b. $n \geq 3$ experiments for each condition, 50,000 cells assayed per experiment. All values analyzed by one-way ANOVA, $p<0.01$. NS denotes $p>0.05$, Tukey's post hoc test

observed between cells treated with $4.75 \mu \mathrm{M}$ and $5 \mu \mathrm{M}$ CCCP: at $4.75 \mu \mathrm{M}$ CCCP, $70 \%$ of cells had either reticular or intermediate morphology, while cells treated with $5 \mu \mathrm{M}$ 
Table 1 Quantitation of mitochondrial morphology in CCCP-treated cells

\begin{tabular}{lccr}
\hline $\mathrm{CCCP}(\mu \mathrm{M})$ & Reticular & Intermediate & Fragmented \\
\hline 0 & $44.4 \pm 17$ & $51.3 \pm 15$ & $4.3 \pm 3$ \\
2 & $39.1 \pm 10$ & $58.9 \pm 11$ & $1.9 \pm 1$ \\
4 & $5.7 \pm 3^{*}$ & $83.5 \pm 6$ & $14.1 \pm 7$ \\
4.25 & $15 \pm 6$ & $69 \pm 4$ & $16 \pm 5$ \\
4.5 & $14.7 \pm 2$ & $72.3 \pm 1$ & $13 \pm 3$ \\
4.75 & $4.7 \pm 1$ & $65 \pm 3$ & $30.3 \pm 3$ \\
5 & $1 \pm 1$ & $19.6 \pm 4^{* *}$ & $79.6 \pm 5^{* *}$ \\
6 & $0 \pm 0$ & $20.5 \pm 8$ & $79.5 \pm 2$ \\
8 & $0 \pm 0$ & $5.6 \pm 2$ & $94.4 \pm 2$ \\
10 & $0 \pm 0$ & $0 \pm 0$ & $100 \pm 0$ \\
\hline
\end{tabular}

Values represent the average percentage of cells with the indicated morphology \pm standard error in three independent experiments, $>150$ cells scored. Statistical analysis: data analyzed by one-way ANOVA, $p<0.05$. $* p<0.05, * * p<0.01$, compared to value immediately above, Tukey's post hoc test. These values were used to generate the bar graph of these data corresponding to Fig. $2 \mathrm{c}$

CCCP showed $80 \%$ fragmented morphology (Fig. 2c; Table 1). Representative images demonstrate this striking threshold: at $4 \mu \mathrm{M} \mathrm{CCCP}$, cells showed extensive regions of mitochondrial interconnection, while at $5 \mu \mathrm{M} \mathrm{CCCP,}$ the mitochondrial network was largely fragmented (Fig. 2d). Similar results were obtained for human HeLa and murine 3T3 cell lines: at $4 \mu \mathrm{M} \mathrm{CCCP}$, both cell lines displayed clear evidence of mitochondrial interconnection when visualized by anti-TOM20 immunolabeling, while HeLa and 3T3 cells treated with $5 \mu \mathrm{M}$ CCCP displayed near-complete fragmentation (Suppl. Figure 1). Strikingly, TMRE fluorescence of WT cells treated with $5 \mu \mathrm{M} \mathrm{CCCP}$ and untreated $\rho^{0}$ cells are statistically equivalent $[1023 \pm 194$ a.u. versus $1197 \pm 197$, respectively, not significant (NS) (Fig. 2b)], and both have fragmented mitochondrial morphology (Figs. 1, 2d). Additional experiments explored whether longer treatments with CCCP alter $\Delta \psi_{\mathrm{m}}$ status: cells incubated with $5 \mu \mathrm{M} \mathrm{CCCP}$ for 1 versus $4 \mathrm{~h}$ showed equivalent TMRE values, as did cells incubated with $10 \mu \mathrm{M} \mathrm{CCCP} \mathrm{(Fig.} \mathrm{2e),} \mathrm{indicating} \mathrm{that}$ increasing CCCP treatment beyond $1 \mathrm{~h}$ does not cause further decreases in $\Delta \psi_{\mathrm{m}}$. This is consistent with findings elsewhere that indicate that protonophores such as CCCP act rapidly to dissipate $\Delta \psi_{\mathrm{m}}$ [41], while longer incubations do not produce additional decreases in $\Delta \psi_{\mathrm{m}}$ [42].

As a Nernstian dye that is taken up by actively respiring mitochondria, TMRE has been extensively used to assay $\Delta \psi_{\mathrm{m}}$ via flow cytometry and quantitative microscopy $[43,44]$. Estimation of $\Delta \psi_{\mathrm{m}}$ from TMRE or other fluorescence methods involves a range of factors, including the concentration of dye within the mitochondria, the cytosol, and the extracellular space, as well as the overall mass of mitochondria within the cell [44]. Moreover, protonophores such as CCCP impact the potential at both the mitochondria and the plasma membrane [45]. Gerencser et al. [46] found that the resting $\Delta \psi_{\mathrm{m}}$ of cultured mammalian cells in highglucose medium is $-139 \mathrm{mV}$, while Springett et al. have successfully employed the oxidation state of mitochondrial bc1 heme groups to quantitatively explore $\Delta \psi_{\mathrm{m}}$, but note the lack of additional direct methods to cross-correlate their measurements [47]. Given these caveats, discussion of $\Delta \psi_{\mathrm{m}}$ levels are here restricted to TMRE fluorescence values, rather than extrapolating these differences to $\mathrm{mV}$. TMRE values are normalized to untreated WT cells (Fig. 2c) to reflect the relative change between samples. Assuming this value as $100 \%$, our results show that $4.75 \mu \mathrm{M} \mathrm{CCCP,} 5 \mu \mathrm{M}$ CCCP, and $\rho^{0}$ cells have relative TMRE levels of 34, 25 and $29 \%$, respectively. These results indicate that both pharmacological and genetic loss of $\Delta \psi_{\mathrm{m}}$ to levels below that of $4.75 \mu \mathrm{M}$-treated WT cells cause dramatic loss of reticular mitochondrial organization, representing a critical threshold required for mitochondrial fission/fusion balance. As mitochondrial dynamics are governed by distinct fission and fusion pathways, we next explored the involvement of OPA1, OMA1, and DRP1 in maintaining $\Delta \psi_{\mathrm{m}}$-dependent mitochondrial dynamic balance.

\section{OMA1-mediated OPA1 cleavage is required for $\Delta \psi_{\mathrm{m}}$-sensitive mitochondrial dynamic balance}

As the mediator of mitochondrial inner membrane fusion, OPA1 has emerged as a key $\Delta \psi_{\mathrm{m}}$-sensitive factor in mitochondrial dynamics. OPA1 exists as five isoforms in human cells (a-e): the a and b long isoforms (L-OPA1) mediate inner membrane fusion, while the $\mathrm{c}, \mathrm{d}$, and e short isoforms (S-OPA1) are fusion-inactive [48]. Dissipation of $\Delta \psi_{\mathrm{m}}$ causes cleavage of fusion-mediating L-OPA1 isoforms to inactive S-OPA1 by the OMA1 protease, causing 
loss of mitochondrial fusion [32, 33], while expression of S-OPA1 activates mitochondrial fission [34]. We, therefore, next explored OPA1 status in $\rho^{0}$ and CCCP-treated 143B models, as in Figs. 1 and 2. Anti-OPA1 Western blotting revealed that WT cells have prominent bands for the L-OPA1 $\mathrm{a}$ and $\mathrm{b}$ isoforms, with the fusion-inactive S-OPA1 showing a strong band for the $\mathrm{d}$ short isoform and minor bands for the $c$ and e isoforms (Fig. 3a). $\rho^{0}$ cells show dramatically decreased L-OPA1 isoforms, with increased levels of the $\mathrm{c}$ and e S-OPA1 isoforms. CCCPtreated WT cells show even more pronounced loss of L-OPA1 isoforms, with a near-complete loss of the a isoform and only a faint band for the $b$ isoform, while the S-OPA1 isoforms are increased, with a major band for the e short isoform (Fig. 3a). Quantitation of OPA1 isoforms confirms this: untreated WT cells maintain $43 \pm 3 \%$ L-OPA1 of total OPA1 signal, while $\rho^{0}$ cells $(33 \pm 2 \%$ L-OPA1) and $10 \mu \mathrm{M}$ CCCP-treated WT cells $(20 \pm 3 \%)$, show significant decreases in the proportion of L-OPA1 relative to untreated WTs. OPA1 blotting at intermediate CCCP concentrations mirrors the morphological threshold observed in Fig. 2c, d: WT cells treated with 4.25, 4.5, and $4.75 \mu \mathrm{M}$ CCCP maintain similar L-OPA1 levels to untreated WT cells $(45 \pm 2,44 \pm 5$, and $46 \pm 4 \%$, respectively), while WT cells treated with $5 \mu \mathrm{M}$ CCCP have a significantly lower proportion of L-OPA1 $(33 \pm 3 \%)$ (Fig. 3b). These results suggest that $>40 \%$ L-OPA1 (of total OPA1) is necessary for mitochondrial fusion capability, and further suggests that the $\Delta \psi_{\mathrm{m}}$ threshold observed for mitochondrial interconnection (Fig. 2) reflects the available pool of fusion-active L-OPA1 isoforms.

As the OMA1 metalloprotease has emerged as a critical regulator of stress-sensitive OPA1-mediated mitochondrial fusion, we next examined the role of OMA1 in this process. While OPA1 is cleaved by a variety of proteases including YME1 [48-50], AFG3L1, and AFG3L2 [33], only OMA1 has been shown to mediate $\Delta \psi_{\mathrm{m}}$-sensitive cleavage of OPA1 [32, 33], suggesting OMA1 as a likely regulator of the observed $\Delta \psi_{\mathrm{m}}$ threshold of mitochondrial fission/fusion balance. To explore this, experiments examined pharmacological and genetic $\Delta \psi_{\mathrm{m}}$ loss, as above, in mouse embryonic fibroblasts (MEFs) either carrying $\left(O M A 1^{+/+}\right)$ or ablated for $\left(O M A 1^{-1-}\right)$ the OMAl gene (kind gift of Dr. Carlos Lopez-Otin, University of Oviedo). When assayed via TMRE flow cytometry, $O M A^{+/+}$cells show an average fluorescence of $2298 \pm 294$ a.u. Strikingly, $O M A 1^{-1-}$ cells show a significantly decreased TMRE signal of $1227 \pm 132$ a.u., relative to $O M A I^{+/+}$cells (Fig. 3c), indicating that these cells have a low basal $\Delta \psi_{\mathrm{m}}$. Both $\mathrm{OMAI}^{+/+}$and $\mathrm{OMAI}^{-/-}$cell lines showed massive decreases in TMRE fluorescence when treated with $20 \mu \mathrm{M}$ CCCP, with average fluorescence values of $567 \pm 50$ and
$544 \pm 21$, respectively (Fig. 3c). Using EtBr treatment per King and Attardi [36], $\rho^{0}$ MEF lines were generated in both $O M A I^{+/+}$and $O M A 1^{-/-}$backgrounds. In both cases, the $\rho^{0}$ line had a dramatically decreased $\Delta \psi_{\mathrm{m}}$ relative to the control MEFs, as assayed by TMRE flow cytometry: $O M A 1^{+/+} \rho^{0}$ cells had average TMRE fluorescence of $925 \pm 42$ versus $2298 \pm 294$ a.u. for control $O M A I^{+/+}$ cells, while $O M A 1^{-1-} \rho^{0}$ cells had TMRE signal of $503 \pm 118$ a.u. versus $1227 \pm 132$ for control $O M A 1^{-1-}$ cells (Fig. 3c). We next examined OPA1 status in $\mathrm{OMAl}^{+/}$ ${ }^{+}$and $O M A I^{-1-}$ cells in the absence or presence of CCCP. While untreated $\mathrm{OMAI}^{+/+}$and $O M A I^{-/-}$cells showed equivalent levels of L- and S-OPA1 isoforms, CCCPtreated $\mathrm{OMAl}^{+/+}$cell lysates showed loss of L-OPA1 isoforms and concomitant increase in S-OPA1 isoforms. Conversely, $\mathrm{OMAl}^{-/-}$cells treated with CCCP showed no change in OPA1 isoforms versus untreated $O M A 1^{-1-}$ control cell lysates (Fig. 3d).

When examined by anti-TOM20 immunolabeling, $O M A 1^{+/+}$MEFs show the expected mix of fragmented and reticular morphologies (Fig. 3d). Conversely, OMAI ${ }^{-/-}$ cells show extensive interconnection (Fig. 3d), indicating that cells lacking the OMA1 metalloprotease retain full mitochondrial fusion capability, despite a low basal $\Delta \psi_{\mathrm{m}}$. When treated with $\mathrm{CCCP}, O M A 1^{+/+}$cells show the expected fragmented morphology, while $O M A 1^{-/-}$cells retain extensive mitochondrial interconnection in the presence of CCCP (Fig. 3d), consistent with findings elsewhere [51, 52]. Similarly, $O M A 1^{+/+} \rho^{0}$ cells show a fragmented morphology, consistent with the results in Figs. 1 and 2, and previous findings [16, 18]. Strikingly, however, $O M A 1^{-/-} \rho^{0}$ cells show extensive mitochondrial interconnection (Fig. 3d), despite the loss of $\Delta \psi_{\mathrm{m}}$. Quantitation of morphology confirms this: while $O M A 1^{+/+}$cells show dramatic loss of reticular and intermediate morphologies when treated with $\mathrm{CCCP}$, compared with untreated $O M A^{+/+}$cells, $O M A 1^{-/-}$cells do not show a significant change in the proportion of cells with reticular or intermediate morphologies when treated with CCCP (Fig. 3f). These results demonstrate that cells lacking OMA1 are insensitive to loss of $\Delta \psi_{\mathrm{m}}$, retaining a fused mitochondrial network when challenged with either pharmacological or genetic loss of $\Delta \psi_{\mathrm{m}}$. However, we note that CCCP-treated $O M A 1^{-1-}$ cells show an overall decrease in the proportion of cells' reticular mitochondria $(21 \pm 13$ versus $40 \pm 3 \%$ for untreated $O M A 1^{-/-}$cells) (Fig. 3f). While this decrease is not statistically significant, it does suggest that additional factors may be involved.

These findings strongly indicate that L-OPA1 is a key determinant of the $\Delta \psi_{\mathrm{m}}$-dependent mitochondrial fission/fusion dynamics observed in Fig. 2. To explore this further, we incubated WT cells with 5 and $10 \mu \mathrm{M} \mathrm{CCCP}$, as in Fig. 2, followed by recovery in CCCP-free media, and 
A

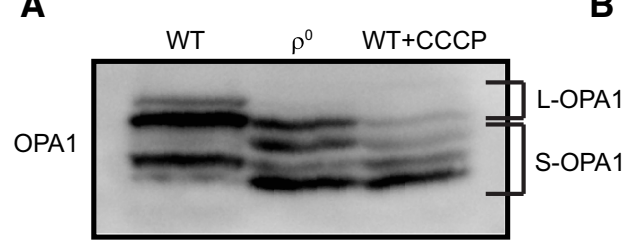

C

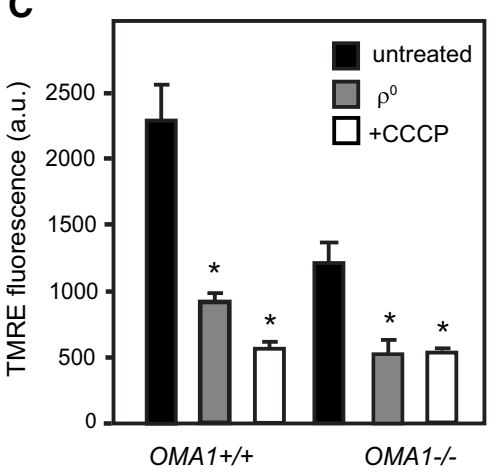

D

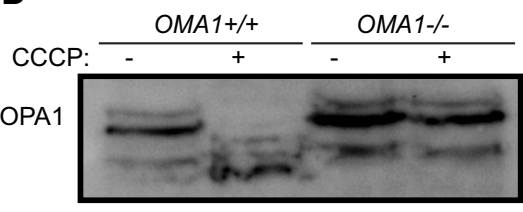

$\mathbf{F}$

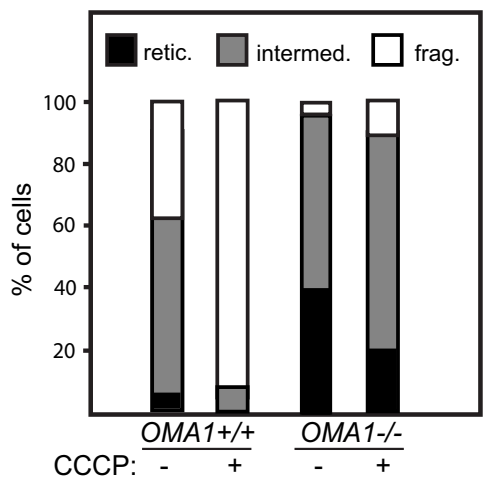

Fig. 3 Role of OPA1 and OMA1 in $\Delta \psi_{\mathrm{m}}$-dependent mitochondrial dynamics. a Anti-OPA1 Western blotting of 143B cells. Lysates were prepared from WT, $\rho^{0}$, and $10 \mu \mathrm{M}$ CCCP-treated WT cells, followed by blotting with anti-OPA1 monoclonal antibody. L-OPA1 $(\mathbf{a}, \mathbf{b})$ and S-OPA1 (c-e) isoforms labeled as indicated. $n=3$ experiments. b Quantitation of Western blot densitometry using Image $\mathrm{J}, \pm \mathrm{SE}$. $n=3$ experiments. *Statistical significance, $p<0.05$, one-way ANOVA followed by Dunnett's post hoc test. c TMRE analysis of $O M A 1^{+/+}$and $O M A 1^{-/-}$mouse embryonic fibroblasts (MEFs). MEFs with $\left(O M A 1^{+/+}\right)$and lacking $\left(O M A 1^{-/-}\right)$the murine OMAl gene were incubated in the absence or presence of $20 \mu \mathrm{M} \mathrm{CCCP}$ for $1 \mathrm{~h}$, or in the absence or presence of EtBr for 10 days. Untreated, CCCPtreated, or $\rho^{0}$ MEFs were incubated with $100 \mathrm{nM}$ TMRE and TMRE fluorescence quantitated via flow cytometry. Average TMRE fluorescence expressed in a.u. \pm SE. $n \geq 3$ experiments for each condition, 50,000 cells assayed per experiment. *Statistical
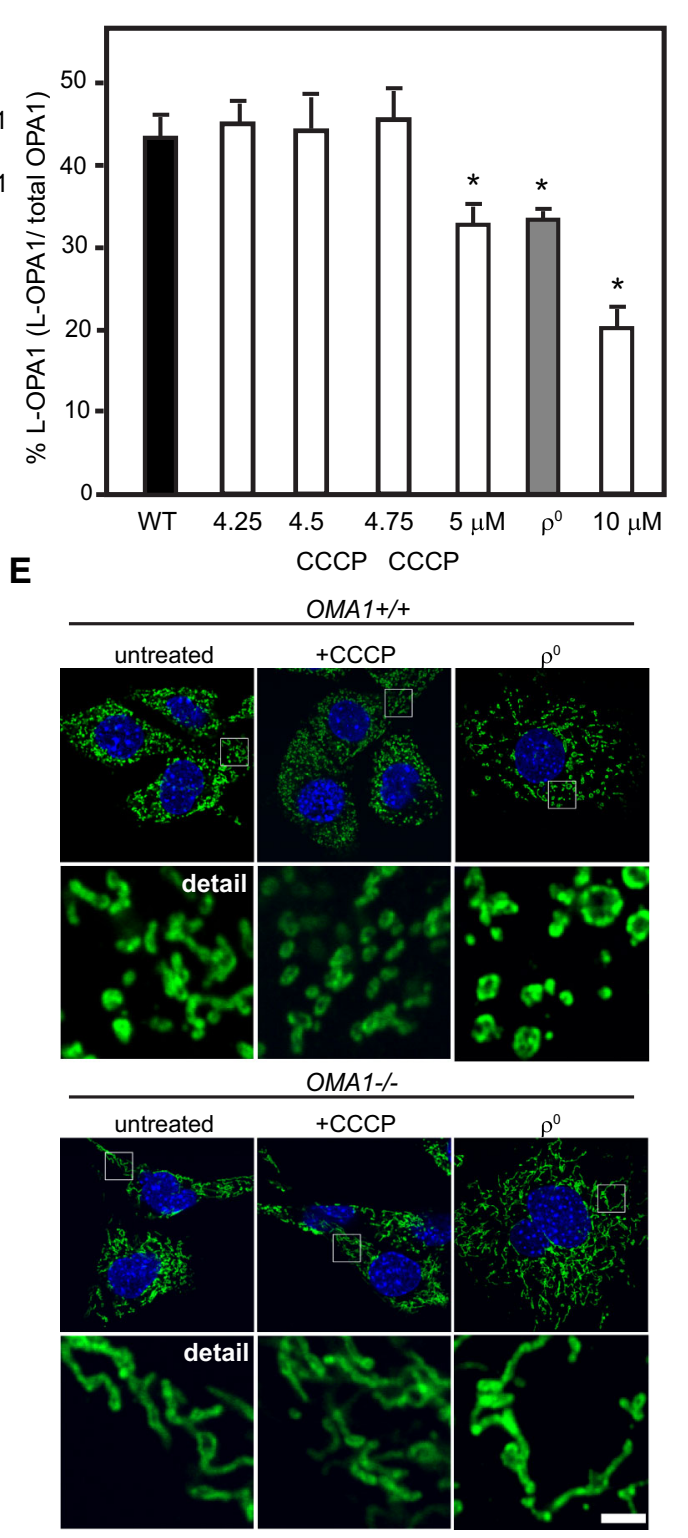

significance, $p<0.01$, one-way ANOVA followed by Dunnett's post hoc test. d Anti-OPA1 Western blotting of lysates from $O M A 1^{+1}$ + and $^{-/-}$MEFs. MEFs were incubated in the absence or presence of $20 \mu \mathrm{M} \mathrm{CCCP}$ for $1 \mathrm{~h}$ and lysates were prepared, followed by blotting to PVDF and blotting with anti-OPA1. $n=3$ experiments. e Mitochondrial morphology of $O M A 1^{+/+}$and $O M A 1^{-/-}$MEFs. Untreated, CCCP-treated $(20 \mu \mathrm{M}, 1 \mathrm{~h})$, or mtDNA-depleted $\rho^{0}$ MEFs in each background were immunolabeled for mitochondrial TOM20 (green) and counterstained with DAPI (blue). $n=3$ experiments. Size bar $2 \mu \mathrm{m}$. f Quantitation of mitochondrial morphology $O M A 1^{+/+}$and $O M A 1^{-1-}$ MEFs in the absence or presence of CCCP. As in Fig. 2c, cells were imaged and scored as having predominantly reticular (black), predominantly fragmented (white), or intermediate (gray) mitochondrial morphology. Average $\%$ of cells with indicated morphology in $n=3$ experiments 
examined mitochondrial morphology. Untreated WT cells maintained the expected balance of mitochondrial fission and fusion, while cells incubated with 5 and $10 \mu \mathrm{M} \mathrm{CCCP}$ both showed the extensive mitochondrial fragmentation noted in Fig. 2 (Fig. 4a). When CCCP-treated cells (either 5or $10 \mu \mathrm{M}$-treated) were allowed to recover in fresh media lacking CCCP, extensive mitochondrial interconnection was observed in both, with highly elongated, networked mitochondria observed (Fig. 4a). Western blotting of CCCPtreated cells showed the expected loss of L-OPA1 isoforms, while CCCP-treated cells allowed to recover in CCCP-free media failed to restore L-OPA1 (Fig. 4b), consistent with the results of Griparic et al. [48]. Thus, while the results of Fig. 3 indicate that L-OPA1 levels correlate with the observed fusion threshold, with OMA1 playing a major role in mediating $\Delta \psi_{\mathrm{m}}$-dependent mitochondrial dynamic balance, Fig. 4 shows that extensive mitochondrial interconnection can nevertheless be demonstrated in the absence of an abundant pool of fusion-active L-OPA1. Similarly, CCCPtreated $O M A 1^{-/-}$cells show a decreased (though not statistically significant) percentage of cells with reticular morphology, relative to untreated $O M A 1^{-/-}$cells (Fig. 3f). Taken together, these findings suggest that other factors likely play a role in $\Delta \psi_{\mathrm{m}}$-dependent mitochondrial dynamic balance. Notably, DRP1 is actively recruited to mitochondria of CCCP-treated cells [53], suggesting that CCCP treatment and recovery cause effective inhibition of DRP1-mediated fission, causing the observed extensive mitochondrial interconnection in Fig. 4. We, therefore, next explored the contribution of DRP1 to $\Delta \psi_{\mathrm{m}}$-dependent mitochondrial dynamic balance.

\section{DRP1 is required for $\Delta \psi_{\mathrm{m}}$-dependent mitochondrial dynamic balance}

To further explore the mechanism of $\Delta \psi_{\mathrm{m}}$-dependent mitochondrial dynamics, we next tested the role of DRP1mediated fission in this process. Western blotting for DRP1 in WT, $\rho^{0}$, and CCCP-treated WT cell lysates revealed no appreciable differences in DRP1 expression between the different cell lines (Fig. 5a). To explore the functional role of DRP1 in $\Delta \psi_{\mathrm{m}}$-sensitive fission/fusion balance, we examined $\Delta \psi_{\mathrm{m}}$ loss under both pharmacological inhibition and genetic knockout of DRP1. Mitochondrial morphology was examined in WT and $\rho^{0}$ cells in the presence or absence of mdivi-1, a small molecular agent that prevents the oligomerization of DRP1 at the mitochondrial outer membrane [13]. Untreated WT cells displayed the expected mixture of fusion and fission, while many mdivi-1-treated WT cells showed elaborately interconnected mitochondria, as visualized using MitoTracker (Suppl. Figure 2A). Quantitation of morphology, as in Fig. 2, reflected this: $36 \pm 3 \%$ of untreated WT cells had a predominantly reticular morphology, but mdivi-1 treatment significantly increased this to $56 \pm 6 \%$. Similarly, the proportion of cells with fragmented mitochondria significantly decreased, falling from $11 \pm 1 \%$ in untreated WT cells to $4 \pm 2 \%$ in response to mdivi-1 (Suppl. Figure 2B). These
Fig. 4 CCCP treatment and recovery restores mitochondrial interconnection. a WT cells were incubated with either 5 or $10 \mu \mathrm{M}$ CCCP for $1 \mathrm{~h}$ without or with subsequent recovery in media lacking CCCP (wash) for $3.5 \mathrm{~h}$ [48] followed by antiTOM20 immunolabeling, $n=3$ experiments. b Western blotting of WT cells treated as in a. Lysates were prepared from untreated WT, $10 \mu \mathrm{M}$ CCCPtreated WT cells, and $10 \mu \mathrm{M}$ CCCP-treated WT cells allowed to recover in CCCP-free media, followed by blotting with antiOPA1 and anti-tubulin monoclonal antibodies. L-OPA1 (a, b) and S-OPA1 (c-

e) isoforms labeled as indicated. $n=3$ experiments

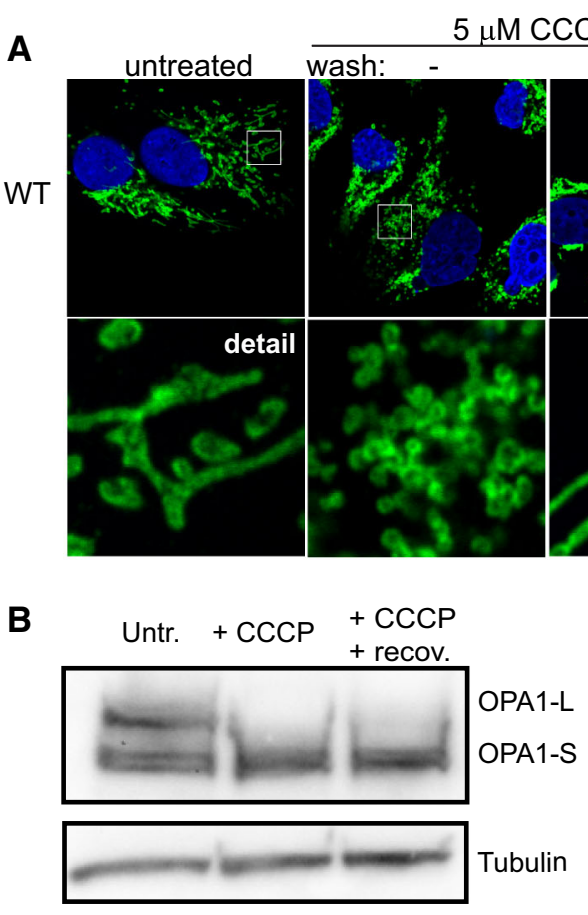


A

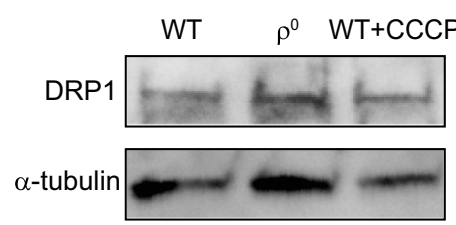

B

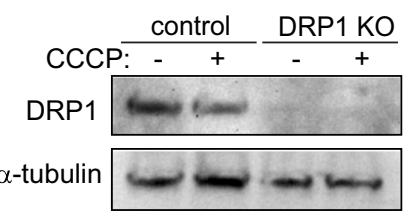

C

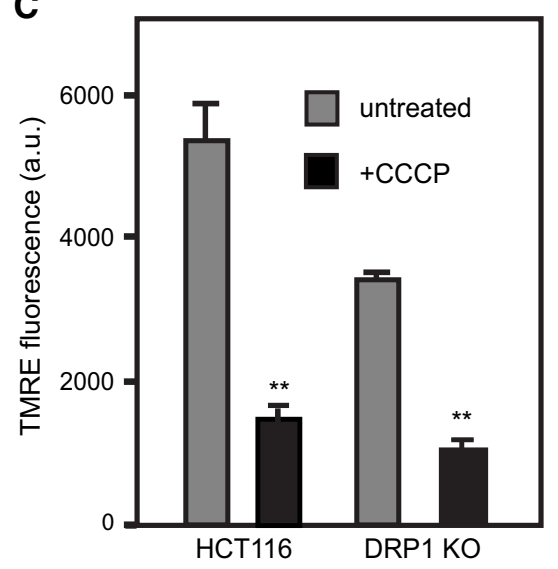

D

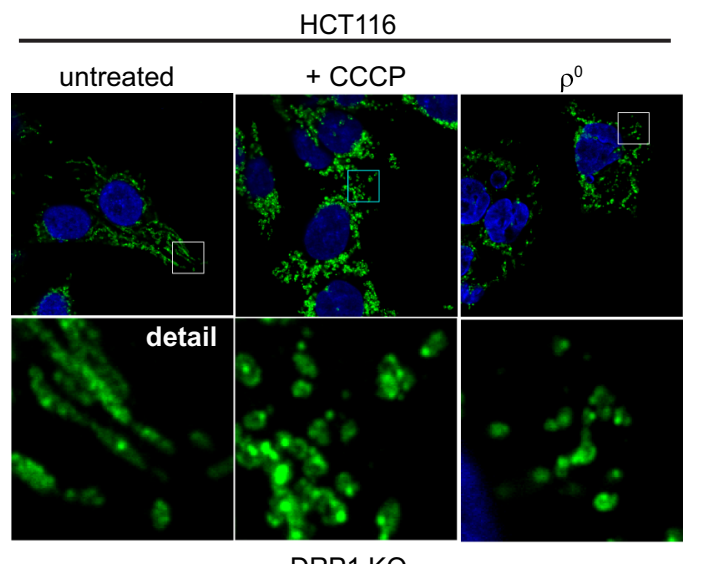

DRP1 KO

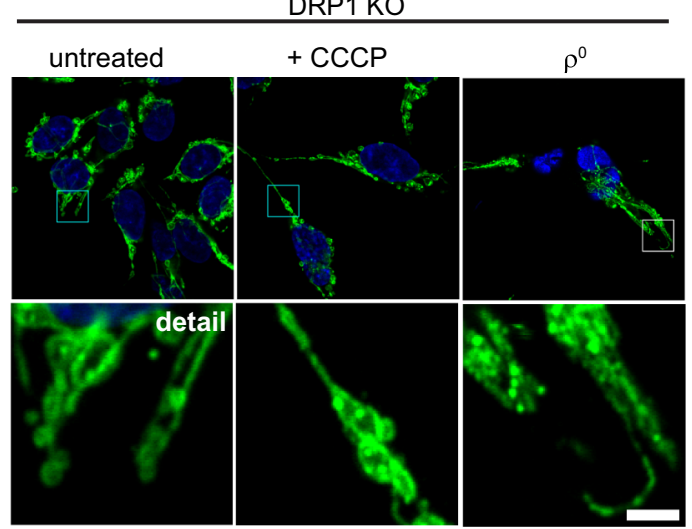

E

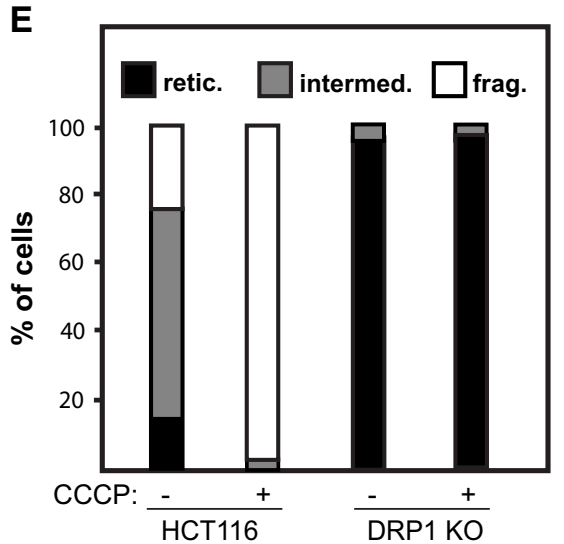

Fig. $5 \Delta \psi_{\mathrm{m}}$-sensitive mitochondrial dynamics require DRP1. a AntiDRP1 Western blotting of lysates from cultured 143B cell lines. Anti$\alpha$-tubulin provides loading control. $n=3$ experiments. b Anti-DRP Western blotting of control and DRP1 knockout HCT116 cells without or with CCCP treatment $(10 \mu \mathrm{M}, 1 \mathrm{~h})$. Anti- $\alpha$-tubulin provides loading control. $n=3$ experiments. c TMRE flow cytometry of HCT116 and DRP1 knockout cell lines in the absence or presence of CCCP $(10 \mu \mathrm{M}, 1 \mathrm{~h})$. Average TMRE fluorescence expressed in a.u. \pm SE. $n \geq 3$ experiments for each condition, 50,000 cells assayed per experiment. **Statistical significance from corresponding untreated cell lines, $p<0.01$, Tukey's post hoc test following one-

results demonstrate that mdivi-1 inhibits fission in this system, causing increased mitochondrial fusion. Untreated $\rho^{0}$ cells show a completely fragmented mitochondrial

way ANOVA. d Immunofluorescence of HCT116 control and DRP1 knockout cells. Untreated, CCCP-treated (10 $\mu \mathrm{M}, 1 \mathrm{~h})$, and mtDNAdepleted $\rho^{0}$ cells in each background were immunolabeled for TOM20 (green) and stained with DAPI (blue). Outlined boxes (white) enlarged in Detail image. Size bar $2 \mu \mathrm{m} . n=3$ experiments. e Quantitation of mitochondrial morphology in HCT116 and DRP1 knockout cells in the absence or presence of CCCP. Cells were imaged and scored as having predominantly reticular (black), predominantly fragmented (white), or intermediate (gray) mitochondrial morphology, as above (Figs. 2c, 3f). Average \% of cells with indicated morphology in $n=3$ experiments

ultrastructure, as in Fig. 1. Strikingly, however, mdivi-1treated $\rho^{0}$ cells also show a completely fragmented morphology, confirmed by quantitation: no $\rho^{0}$ cells were 
observed to have a reticular morphology in either the absence or presence of mdivi-1, (Suppl. Figure 2A, B). Similar results were obtained in CCCP-treated WT cells: mdivi-1-treated WT cells challenged with 5 or $10 \mu \mathrm{M}$ CCCP also showed total fragmentation of the mitochondrial network, with no observable organellar interconnection (Suppl. Figure 2C), suggesting that $\Delta \psi_{\mathrm{m}^{-}}$ dependent fragmentation of the mitochondrial network might be independent of DRP1-mediated fission.

However, mdivi-1 may not be sufficiently potent to completely inhibit DRP1 fission activity, particularly if loss of $\Delta \psi_{\mathrm{m}}$ stimulates DRP1 functional activity, as found elsewhere [39] via phosphorylation at residue Ser656 [54]. To more rigorously test the requirement for DRP1 in $\Delta \psi_{\mathrm{m}^{-}}$ sensitive mitochondrial dynamics, we examined human HCT116 cells containing or knocked out for DRP1 (kind gift of Dr. Richard Youle, NIH) with pharmacological (CCCP) or genetic (mtDNA-depleted) loss of $\Delta \psi_{\mathrm{m}}$. AntiDRP1 Western blotting of HCT116 control and DRP1 knockout cells confirmed that DRP1 is present in both untreated and CCCP-treated HCT116 cells, while DRP1 knockout cells (without or with CCCP treatment) lack DRP1 (Fig. 5b). TMRE flow cytometry was used as above to monitor $\Delta \psi_{\mathrm{m}}$ : HCT116 cells maintained a TMRE signal of $5368 \pm 551$ a.u. while CCCP treatment lowered their TMRE value to $1438 \pm 230$ a.u. DRP1 knockout cells, however, had an average TMRE fluorescence of $3379 \pm 85$ a.u., significantly lower than control HCT116s, consistent with bioenergetic functional defects in cells with genetically altered fission or fusion dynamics [55]. CCCPtreated DRP1 knockout cells showed a dramatic decrease in TMRE signal to $1092 \pm 72$ a.u., confirming that CCCP dissipates $\Delta \psi_{\mathrm{m}}$ in HCT116 and DRP1KO cells (Fig. 5c). Mitochondrial morphology of HCT116 and DRP1KO cells was monitored via confocal microscopy with anti-TOM20 immunolabeling, as above. While control HCT116 cells maintained a largely intermediate morphology, with regions of both mitochondrial interconnection and fragmentation, DRP1 knockout cells showed elaborately interconnected mitochondria, as visualized by TOM20 imaging (Fig. 5d). In response to either CCCP treatment or depletion of mtDNA (via EtBr treatment), control HCT116 cells show fragmentation of the mitochondrial network, as in Fig. 1. Conversely, DRP1 knockout cells maintain an extensively interconnected mitochondrial network when treated with CCCP or under mtDNA depletion (Fig. 5d). Quantitation of morphology, as in Figs. 2c and 3f, supports this: CCCP-treated HCT116 cells show a dramatic increase in cells with fragmented mitochondria $(98 \pm 1 \%)$ compared with untreated HCT116s $(24 \pm 6 \%)$. Conversely, DRP1 knockout cells show overwhelmingly reticular morphology $(96 \pm 2 \%)$ that does not change in response to CCCP treatment $(97 \pm 2 \%)$. These results demonstrate that DRP1 knockout cells are insensitive to loss of $\Delta \psi_{\mathrm{m}}$, and indicate that DRP1 plays a major role in maintaining $\Delta \psi_{\mathrm{m}}$-dependent mitochondrial fission/fusion balance.

\section{DRP1 and OMA1 coordinately impact mitochondrial fusion and OPA1}

Collectively, our findings indicate that both OMA1 and DRP1 are required for $\Delta \psi_{\mathrm{m}}$-dependent mitochondrial dynamic balance, as cells lacking either factor maintain mitochondrial interconnection when challenged with loss of $\Delta \psi_{\mathrm{m}}$ (Figs. 3, 5). Moreover, WT cells challenged with CCCP followed by recovery in CCCP-free media show extensive reticular mitochondria, despite the lack of abundant L-OPA1 in these cells (Fig. 4). These findings suggest that OMA1 and DRP1 cooperatively act to mediate $\Delta \psi_{\mathrm{m}}$-dependent mitochondrial fusion/fission balance.

To explore this possibility, we hypothesized that CCCP challenge and recovery would cause an additive increase in mitochondrial interconnection even in the presence of a stable pool of L-OPA1. To test this, we challenged $O M A I^{-1-}$ cells with CCCP treatment and recovery (in CCCP-free media) as in Fig. 4. Untreated $O M A 1^{-1-}$ cells showed the expected mixture of reticular and intermediate morphologies expected, as per Fig. 3. Strikingly, however, $O M A 1^{-1-}$ cells challenged with CCCP and recovery showed a robust increase in reticular mitochondrial morphology, with elaborately interconnected mitochondria apparent (Fig. 6a). Quantitation confirmed this: while $28 \pm 2 \%$ of untreated $O M A I^{-/-}$cells had a reticular mitochondrial morphology, OMA1 ${ }^{-/-}$cells given $\mathrm{CCCP}$ challenge and recovery showed a significant increase, with $45 \pm 5 \%$ of cells showing a reticular mitochondrial morphology. Thus, despite the presence of a stable pool of

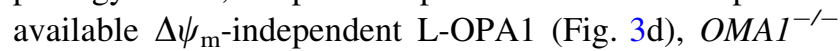
cells show an additive increase in mitochondrial interconnection when challenged with $\mathrm{CCCP}$ and recovery (Fig. 6a, b), strongly indicating that DRP1 and OMA1 together modulate mitochondrial dynamic balance. While OMA1 has been extensively characterized as a key mediator of $\Delta \psi_{\mathrm{m}}$-dependent OPA1 cleavage [32-34], we examined OPA1 status in DRP1 knockout and control cells to see whether DRP1 impacts OPA1.

To explore this, we examined HCT116 control and DRP1 knockout cells via OPA1 Western blotting. Control HCT116 cells show the expected distribution of OPA1 isoforms, with prominent bands for the $\mathrm{b}$ and $\mathrm{d}$ isoforms. Upon incubation with CCCP, HCT116 cells show cleavage of L-OPA1 isoforms, resulting in accumulation of S-OPA1 isoforms (Fig. 6c), consistent with the mitochondrial fragmentation found in CCCP-treated HCT116s in Fig. 5d. Untreated DRP1 knockout cells show the five OPA1 isoforms, with comparatively lower L-OPA1 levels than 
A

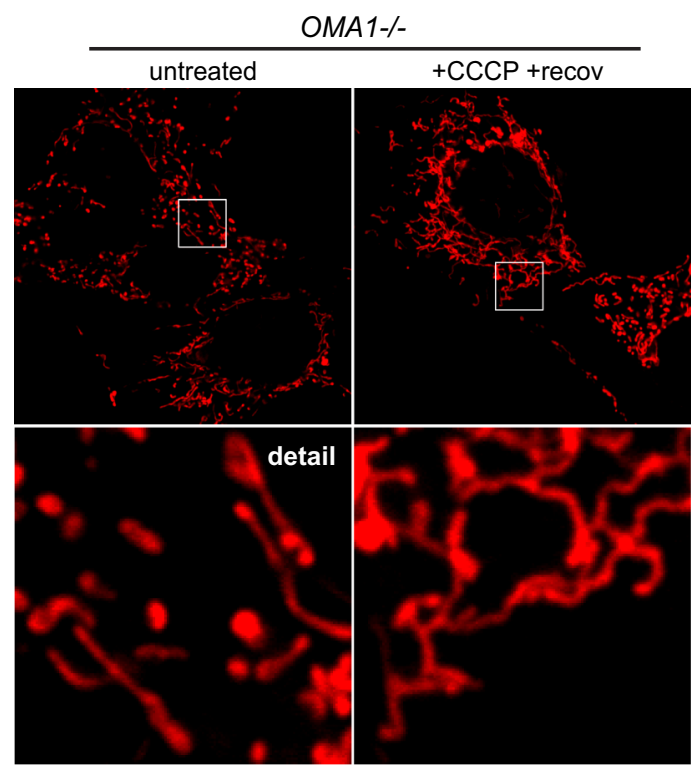

C

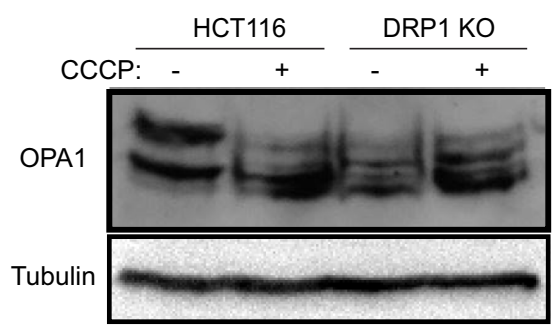

D

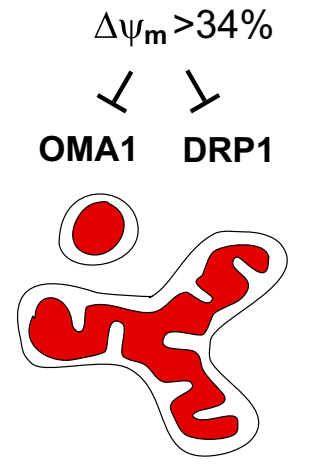

$\Delta \psi_{\mathrm{m}}<34 \%$
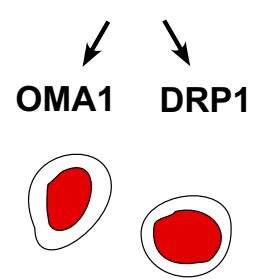

B

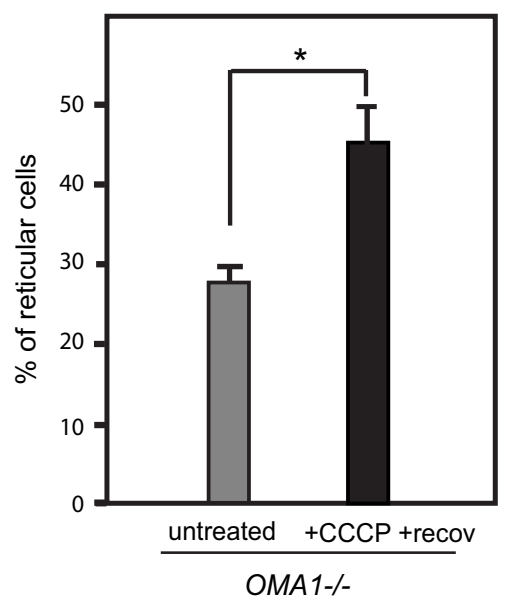

Fig. 6 Cooperative roles for OMA1 and DRP1 in $\Delta \psi_{\mathrm{m}}$-dependent mitochondrial dynamics. a Untreated and CCCP-treated + recovery $O M A 1^{-1-}$ cells were incubated with MitoTracker and visualized by confocal microscopy, $n=3$ experiments. b Quantitation of mitochondrial morphology of cells in a. Percentage of cells with predominantly reticular morphology in untreated versus CCCPtreated + recovery $O M A 1^{-/-}$cells, $n=3$ experiments. ${ }^{*}$ Statistical significance, $p<0.01$, Student's $t$ test. c Anti-OPA1 blotting of control and DRP1 knockout HCT116 cells. Cells were incubated in the absence or presence of $10 \mu \mathrm{M} \mathrm{CCCP}$ for $1 \mathrm{~h}$ and lysates were

control HCT116 cells. Strikingly, however, DRP1 knockout cells treated with CCCP show no appreciable change in OPA1 status: L- and S-OPA1 levels are essentially identical in control versus CCCP-treated DRP1 knockout cells. This is consistent with the extensive mitochondrial interconnection shown in CCCP-treated DRP1 knockouts prepared, followed by blotting with anti-OPA1. $n=3$ experiments. d Model of $\Delta \psi_{\mathrm{m}}$-dependent fission/fusion balance. An intact $\Delta \psi_{\mathrm{m}}$ (at or above the $34 \%$ TMRE threshold) acts to inhibit both L-OPA1 cleavage by OMA1 and recruitment of DRP1 to mitochondria, resulting in intact mitochondrial fusion. When $\Delta \psi_{\mathrm{m}}$ falls below the observed threshold, fission is activated via increased DRP recruitment to the mitochondria, while OMA1 proteolytic activity is activated, rapidly depleting L-OPA1, resulting in a completely fragmented population of individual organelles

(Fig. 5d, e). While DRP1 knockouts have decreased L-OPA1 relative to control HCT116s, they do not show any activation of OPA1 cleavage in response to $\mathrm{CCCP}$ treatment (Fig. 6c). These findings are consistent with loss of L-OPA1 in response to DRP1 downregulation [56], and suggest that the DRP1-mediated fission machinery interacts 
with OPA1 and the mitochondrial fusion factors to cooperatively mediate $\Delta \psi_{\mathrm{m}}$-dependent mitochondrial dynamic balance.

\section{Discussion}

The interaction of bioenergetic function with fusion and fission pathways drives the structure/function dynamics of mitochondria as an organellar network. While it has been shown that complete dissipation of $\Delta \psi_{\mathrm{m}}$ causes mitochondrial fragmentation, we here examine (1) the level of $\Delta \psi_{\mathrm{m}}$ required, and (2) the contribution of both fission and fusion pathways, in maintaining $\Delta \psi_{\mathrm{m}}$-dependent mitochondrial dynamic balance in human cells. Our findings indicate that a threshold of 34\% TMRE-assayed $\Delta \psi_{\mathrm{m}}$, strongly correlating with available fusion-active L-OPA1, is required to maintain mitochondrial fission/fusion balance. Surprisingly, cells lacking either OMA1 or DRP1 have obligate mitochondrial fusion and fail to cleave long OPA1 isoforms in response to pharmacological or genetic loss of $\Delta \psi_{\mathrm{m}}$. These results suggest that OMA1 and DRP1 cooperatively act to mediate mitochondrial $\Delta \psi_{\mathrm{m}}$-dependent dynamic balance, and provide further evidence in support of a proposed mechanistic link between DRP1-mediated fission and OPA1 [34, 57]. As mitochondrial dynamic balance is crucial to cellular processes such as apoptosis, this 'tipping point' mechanism of mitochondrial dynamic balance will have far-reaching impacts on these key pathways.

\section{Mitochondrial dynamic balance requires a threshold of $\Delta \psi_{\mathrm{m}}$}

Here, we address a key question of mitochondrial structure/function relationships: to what extent can the mitochondrial network lose bioenergetic function before dynamic balance is compromised? While total collapse of $\Delta \psi_{\mathrm{m}}$ has been shown to cause fragmentation of the mitochondrial network [4, 32, 33], the functional tipping point of $\Delta \psi_{\mathrm{m}}$-dependent mitochondrial dynamics has remained unknown. Mitochondrial threshold effects have been extensively documented in mtDNA-derived neuromuscular diseases: cells or tissues carrying greater than 80-90\% mutant mtDNA typically show collapse of bioenergetic capacity [30]. Previously, we found that cells carrying a mutation load greater than $90 \% \Delta$-mtDNA lost the ability to maintain mitochondrial interconnection [17]. Here, we explore the functional and mechanistic tipping point behind this threshold using TMRE flow cytometry to assay $\Delta \psi_{\mathrm{m}}$, and confocal imaging to monitor mitochondrial organization. When normalized against the TMRE value of untreated WT cells as $100 \%$, our data indicate that cells below 34\% TMRE signal have disrupted fission/fusion homeostasis. CCCP titration demonstrates that at concentrations above $4.75 \mu \mathrm{M}$, cells have obligate fragmentation of the mitochondrial network (Fig. 2). This loss of mitochondrial interconnection correlates with loss of fusionactive L-OPA1 isoforms (Fig. 3a, b), strongly indicating that OPA1-mediated inner membrane fusion per se is lost beyond this point. These findings demonstrate a key bioenergetic determinant of mitochondrial fission/fusion dynamics. Further research may employ alternate methods such as live-cell imaging [43] and heme oxidation state [47] to confirm the $\Delta \psi_{\mathrm{m}}$ threshold in $\mathrm{mV}$. In addition, this $\Delta \psi_{\mathrm{m}}$ threshold likely contributes to the canonical mitochondrial genetic threshold effect: our model predicts that any mtDNA mutation that pushes $\Delta \psi_{\mathrm{m}}$ below this threshold commits the organelle to obligate fission. As such, this parameter is likely to be of critical importance to mitochondrial pathology in a range of human diseases, as well as therapeutic strategies seeking to eliminate $\Delta \psi_{\mathrm{m}}$-impacting mutant mtDNAs through pathways such as autophagy [58].

\section{OMA1 and DRP1 coordinately mediate $\Delta \psi_{\mathrm{m}^{-}}$ dependent mitochondrial dynamics}

As mitochondrial fission and fusion pathways have distinct sets of mediating factors, either pathway (or both) could be mechanistically responsible for this tipping point threshold. Mitochondrial fusion has been shown to be dependent on $\Delta \psi_{\mathrm{m}}$ [4], mediated by L-OPA1 isoforms [48] that are cleaved to fusion-inactive S-OPA1 by OMA1 in response to loss of $\Delta \psi_{\mathrm{m}}[32,33]$. These findings suggested that fusion is the major mediator of $\Delta \psi_{\mathrm{m}}$-dependent mitochondrial dynamics, somewhat de-emphasizing the role of DRP1-mediated fission. However, our results show that cells lacking either OMA1 or DRP1 are insensitive to loss of $\Delta \psi_{\mathrm{m}}$. If OMA1 alone controlled $\Delta \psi_{\mathrm{m}}$-dependent mitochondrial dynamics, DRP1 knockout cells would fragment in $\rho^{0}$ or CCCP-treated lines. The highly interconnected mitochondrial morphology of DRP1 knockout cells (Fig. 4) despite their lack of abundant L-OPA1 (Fig. 5) demonstrates the importance of DRP1. Similarly, cells treated with CCCP, with subsequent recovery in fresh media, do not show restoration of L-OPA1 (Fig. 4b), but nevertheless show striking mitochondrial interconnection (Fig. 4a). Taken together, these data support a strong role for DRP1 and fission per se in maintaining $\Delta \psi_{\mathrm{m}}$-dependent mitochondrial dynamic balance.

Moreover, our results support both functional coordination and mechanistic interaction of OPA1-mediated fusion and DRP1-mediated fission. While experiments in cells lacking OMA1 (Fig. 3) or DRP1 (Fig. 5) show that both are necessary for mitochondrial dynamic balance, the 
decreased L-OPA1 observed in DRP1 knockout cells (Fig. 6c) indicates that DRP1 is necessary to maintain L-OPA1 stability. This enhanced OPA1 processing is consistent with Mopert et al. [57], who found similar results in response to transient knockdown of DRP1, while Huang et al. found that DRP interacts with mitofusin 2 [59], consistent with DRP1 interactions with the mitochondrial fusion machinery. These findings suggest a role for DRP1 in stabilizing mitochondrial fission/fusion factors via direct and indirect protein-protein interactions spanning the outer and inner mitochondrial membranes.

Thus, while mitochondrial fission and fusion have frequently been discussed as mechanistically distinct pathways, these results support a model in which an intact (i.e., above threshold) $\Delta \psi_{\mathrm{m}}$ provides a check to both OMA1 and DRP1, allowing a balance of fission and fusion with both DRP1 and OMA1 interacting (directly or indirectly) with OPA1. Loss of $\Delta \psi_{\mathrm{m}}$ to levels below the observed 34\% TMRE threshold simultaneously activates OMA1 cleavage of L-OPA1 and mitochondrial recruitment of DRP1, collapsing mitochondrial organization to an obligately fragmented state (Fig. 6d). It is likely that the loss of $\Delta \psi_{\mathrm{m}}$ below threshold causes profound changes in conformation and enzymatic activity of multiple proteins at the mitochondrial inner and outer membranes, altering the protein-protein interactions that determine mitochondrial structural dynamics. OMA1 has been shown to undergo self-cleavage and activation of L-OPA1 proteolysis during membrane depolarization [60], while Anand et al. found that expression of S-OPA1 is sufficient to induce mitochondrial fission, with S-OPA1 frequently colocalizing with DRP1 at ER-mitochondrial sites of contact [34]; the authors postulated that S-OPA1 may stimulate DRP1 fission activity. $\Delta \psi_{\mathrm{m}}$-dependent DRP1 fission activity is activated by phosphorylation at S637 [27], and can be activated by calcineurin in response to increased cytosolic calcium [53]. Recruitment of DRP1 to mitochondria is mediated by actin [61, 62]. Taken together, these findings indicate that $\Delta \psi_{\mathrm{m}}$-dependent mitochondrial structural homeostasis involves the highly dynamic realignment and recruitment of multiple factors in different mitochondrial and cellular compartments.

Mitochondrial fission/fusion status directly impacts a broad range of cellular processes such as apoptosis. Consistent with this, cells below the $\Delta \psi_{\mathrm{m}}$ threshold show dramatically decreased viability when challenged with galactose-containing media (Suppl. Figure 3). As such, this tipping point threshold of $\Delta \psi_{\mathrm{m}}$-dependent fission/fusion balance may have major impacts on cell-wide signaling events including autophagy, mitosis, and nutrient utilization. For example, collapse of $\Delta \psi_{\mathrm{m}}$ is a key step in mitochondrial autophagy [63], causing recruitment of the Parkin E3 ubiquitin ligase to the mitochondria and targeting the organelle to the autophagosome [64], while mitochondrial fission is directly activated by AMP kinase signaling [65]. These findings thus have direct relevance to a wide range of prevalent diseases that include mitochondrial dysfunction in their pathogenesis. Mitochondrial dynamics are increasingly found to be critical for energetically dependent tissues such as heart [66] and skeletal muscle [67], with decreased OxPhos function and disrupted fission/fusion balance emerging in patient samples and disease models of neuromuscular diseases [30], neurodegenerative aging and Parkinson's disease [68, 69], diabetes [70-72], and heart failure [28]. As such, our findings illustrate a basic mechanism of mitochondrial structure/function homeostasis with major implications for the pathogenesis and translational treatment of these disorders.

\section{Materials and methods}

\section{Cell culture}

Human 143B osteosarcoma cell lines FLP6a39.2 (WT) and $143 \mathrm{~B} 206\left(\rho^{0}\right)$ were described previously $[16,19]$, as have $O M A I^{+/+}$and $O M A I^{-/-}$mouse embryonic fibroblasts (MEFs) [52] and human colorectal carcinoma HCT116 control and DRP1 knockout cell lines [73]. Cells were grown in high-glucose Dulbecco's Modified Eagle's medium (DMEM) with $10 \%$ fetal bovine serum supplemented with $50 \mu \mathrm{g} / \mathrm{mL}$ uridine in $5 \% \mathrm{CO}_{2}$ at $37{ }^{\circ} \mathrm{C}$. CCCP and mdivi-1 (Sigma, St. Louis, MO, USA) were dissolved as stock solutions in DMSO for dilution in complete media. All cell culture reagents were from ThermoFisher (Waltham, MA, USA).

\section{Fluorescence microscopy}

Cultured cells were seeded to $18 \mathrm{~mm}^{2}$ glass coverslips. Coverslips were incubated with MitoTracker CMXRos (Invitrogen Molecular Probes, Carlsbad, CA, USA), followed by fixation in $4 \%$ paraformaldehyde in PBS. For immunolabeling of the translocase of the outer mitochondrial membrane-20 protein (TOM20), cells were permeabilized with $0.1 \%$ TX-100 in PBS, followed by blocking in $10 \%$ normal goat serum and incubation with anti-TOM20 monoclonal antibody FL-145 at 1:100 dilution (Santa Cruz Biotechnology, Santa Cruz, CA, USA). Coverslips were incubated with goat anti-mouse Alexa488conjugated secondary antibody at 1:100 dilution (Invitrogen Molecular Probes, Carlsbad, CA, USA), followed by staining with diaminophenylindole (DAPI) and mounting in 50\% glycerol in PBS. Coverslips were imaged on an Olympus Fluoview FV-10i (Olympus, Center Valley, PA, USA) with a $60 \times$ UPLSAP60xW objective with aperture 
1.2 and $3 \times$ optical zoom at room temperature. For scoring of mitochondrial morphology, individual cell profiles on confocal micrographs were scored as predominantly reticular if they had fewer than three instances of fragmented mitochondria. Cells were scored as predominantly fragmented if they displayed fewer than three instances of mitochondrial interconnection. All others were scored as having intermediate mitochondrial morphology.

\section{Flow cytometry}

To assay $\Delta \psi_{\mathrm{m}}$ using flow cytometry, we modified our previous method using the $\Delta \psi_{\mathrm{m}}$-specific dye tetramethyl rhodamine ester (TMRE) [19]. Briefly, cells were seeded in 100-mm dishes and incubated with $100 \mathrm{nM}$ TMRE for $20 \mathrm{~min}$, followed by trypsinization and two washes in PBS. Cells were resuspended in $1 \mathrm{~mL}$ of PBS and analyzed using a BD Biosciences LSR Fortessa.

\section{PCR analysis of mtDNA}

Total cellular DNA was isolated using proteinase $\mathrm{K}$ digestion, followed by phenol/chloroform extraction and ethanol precipitation, as previously [16]. Forward primer ACGCCAAAATCCATTTCACT and reverse primer CGGGAATTGCATCTGTTTTT amplify nt7130-8113 of human mtDNA. Reactions were electrophoresed on a $1 \%$ agarose gel and imaged using a Fotodyne Foto/Eclipse gel documentation system.

\section{Western blot analysis}

Cells were lysed using a modified RIPA buffer on ice, and lysates were run on a 10\% SDS-PAGE gel, followed by transfer to Immobilon PVDF membrane (Bio-Rad, Redmond, WA, USA). Anti-OPA1 blotting used separation of proteins on a $6 \%$ SDS-PAGE gel, per Griparic et al. [48]. Membranes were blocked in 5\% milk in TBS overnight at 4 degrees $\mathrm{C}$, followed by incubation with anti-MTCO2 monoclonal antibody ab110258 at 1:1000 dilution (Abcam, Cambridge, MA, USA), anti-DRP1 monoclonal ab56788 at 1:1000 dilution (Abcam, Cambridge, MA, USA), anti-OPA1 monoclonal antibody 612606 at 1:500 dilution (BD Biosciences, San Jose, CA, USA) and goat anti-mouse polyHRP secondary antibody at 1:3000 dilution. Blots were developed using WestDura chemiluminescent reagents and imaged using a Kodak 4000MM Image Station or Bio-Rad ChemiDoc XRS. Anti- $\alpha$-tubulin monoclonal antibody (Sigma) was employed as a loading control.

Acknowledgements The authors gratefully acknowledge Carlos Lopez-Otin and Pedro Quiros (University of Oviedo) for providing $O M A 1^{+/+}$and $O M A 1^{-/-}$MEFs, Richard Youle (National Institutes of Health) for providing HCT116 control and DRP1 KO cells, and Eric Schon (Columbia University) for providing FLP6a39.2 (WT), FLP6a39.32 $(\Delta)$, and $143 \mathrm{~B} 206 \rho^{0}$ osteosarcoma cells. This work was supported by the National Institutes of Health 1SC3116669-01 and Diabetes Action Research and Education Foundation Grants 378 and 409 (to R.G.), with undergraduate training support from National Institutes of Health 5R25GM100866-03 (for E.J.) and UTPA Science Education Grant \#52007568 from the Howard Hughes Medical Institute (for N.G. and I.G.). D.A. and M.R. were supported by the UTRGV High Scholars Program.

\section{Compliance with ethical standards}

Conflict of interest The authors declare that they have no conflict of interest.

Open Access This article is distributed under the terms of the Creative Commons Attribution 4.0 International License (http:// creativecommons.org/licenses/by/4.0/), which permits unrestricted use, distribution, and reproduction in any medium, provided you give appropriate credit to the original author(s) and the source, provide a link to the Creative Commons license, and indicate if changes were made.

\section{References}

1. Amchenkova AA, Bakeeva LE, Chentsov YS, Skulachev VP, Zorov DB (1988) Coupling membranes as energy-transmitting cables. I. Filamentous mitochondria in fibroblasts and mitochondrial clusters in cardiomyocytes. J Cell Biol 107(2):481-495

2. Chan DC (2012) Fusion and fission: interlinked processes critical for mitochondrial health. Annu Rev Genet 46:265-287

3. Nunnari J, Suomalainen A (2012) Mitochondria: in sickness and in health. Cell 148(6):1145-1159

4. Legros F, Lombes A, Frachon P, Rojo M (2002) Mitochondrial fusion in human cells is efficient, requires the inner membrane potential, and is mediated by mitofusins. Mol Biol Cell 13(12):4343-4354

5. Olichon A, Emorine LJ, Descoins E et al (2002) The human dynamin-related protein OPA1 is anchored to the mitochondrial inner membrane facing the inter-membrane space. FEBS Lett 523(1-3):171-176

6. Olichon A, Baricault L, Gas N et al (2003) Loss of OPA1 perturbates the mitochondrial inner membrane structure and integrity, leading to cytochrome c release and apoptosis. J Biol Chem 278(10):7743-7746

7. Cipolat S, Martins de Brito O, Dal Zilio B, Scorrano L (2004) OPA1 requires mitofusin 1 to promote mitochondrial fusion. Proc Natl Acad Sci USA 101(45):15927-15932

8. Ono T, Isobe K, Nakada K, Hayashi JI (2001) Human cells are protected from mitochondrial dysfunction by complementation of DNA products in fused mitochondria. Nat Genet 28(3):272-275

9. Smirnova E, Griparic L, Shurland DL, van der Bliek AM (2001) Dynamin-related protein Drp1 is required for mitochondrial division in mammalian cells. Mol Biol Cell 12(8):2245-2256

10. Yoon Y, Krueger EW, Oswald BJ, McNiven MA (2003) The mitochondrial protein hFis1 regulates mitochondrial fission in mammalian cells through an interaction with the dynamin-like protein DLP1. Mol Cell Biol 23(15):5409-5420

11. James DI, Parone PA, Mattenberger Y, Martinou JC (2003) hFis1, a novel component of the mammalian mitochondrial fission machinery. J Biol Chem 278(38):36373-36379

12. Gandre-Babbe S, van der Bliek AM (2008) The novel tail-anchored membrane protein Mff controls mitochondrial and 
peroxisomal fission in mammalian cells. Mol Biol Cell 19(6):2402-2412

13. Cassidy-Stone A, Chipuk JE, Ingerman E et al (2008) Chemical inhibition of the mitochondrial division dynamin reveals its role in Bax/Bak-dependent mitochondrial outer membrane permeabilization. Dev Cell 14(2):193-204

14. Cui M, Tang X, Christian WV, Yoon Y, Tieu K (2010) Perturbations in mitochondrial dynamics induced by human mutant PINK1 can be rescued by the mitochondrial division inhibitor mdivi-1. J Biol Chem 285(15):11740-11752

15. Mishra P, Chan DC (2016) Metabolic regulation of mitochondrial dynamics. J Cell Biol 212(4):379-387

16. Gilkerson RW, Schon EA, Hernandez E, Davidson MM (2008) Mitochondrial nucleoids maintain genetic autonomy but allow for functional complementation. J Cell Biol 181(7):1117-1128

17. Santra S, Gilkerson RW, Davidson M, Schon EA (2004) Ketogenic treatment reduces deleted mitochondrial DNAs in cultured human cells. Ann Neurol 56(5):662-669

18. Gilkerson RW, Margineantu DH, Capaldi RA, Selker JM (2000) Mitochondrial DNA depletion causes morphological changes in the mitochondrial reticulum of cultured human cells. FEBS Lett 474(1):1-4

19. Gilkerson RW, De Vries RL, Lebot P et al (2012) Mitochondrial autophagy in cells with mtDNA mutations results from synergistic loss of transmembrane potential and mTORC1 inhibition. Hum Mol Genet 21(5):978-990

20. Malka F, Guillery O, Cifuentes-Diaz C et al (2005) Separate fusion of outer and inner mitochondrial membranes. EMBO Rep 6(9):853-859

21. Yang L, Long Q, Liu J et al (2015) Mitochondrial fusion provides an 'initial metabolic complementation' controlled by mtDNA. Cell Mol Life Sci 72(13):2585-2598

22. Katajisto P, Dohla J, Chaffer CL et al (2015) Stem cells. Asymmetric apportioning of aged mitochondria between daughter cells is required for stemness. Science 348(6232):340-343

23. Kashatus DF, Lim KH, Brady DC et al (2011) RALA and RALBP1 regulate mitochondrial fission at mitosis. Nat Cell Biol 13(9):1108-1115

24. Taguchi N, Ishihara N, Jofuku A, Oka T, Mihara K (2007) Mitotic phosphorylation of dynamin-related GTPase Drp1 participates in mitochondrial fission. $J$ Biol Chem 282(15):11521-11529

25. Youle RJ, Karbowski M (2005) Mitochondrial fission in apoptosis. Nat Rev Mol Cell Biol 6(8):657-663

26. Twig G, Elorza A, Molina AJ et al (2008) Fission and selective fusion govern mitochondrial segregation and elimination by autophagy. EMBO J 27(2):433-446

27. Rambold AS, Kostelecky B, Elia N, Lippincott-Schwartz J (2011) Tubular network formation protects mitochondria from autophagosomal degradation during nutrient starvation. Proc Natl Acad Sci USA 108(25):10190-10195

28. Dorn GW, $2^{\text {nd }}$ (2015) Mitochondrial dynamism and heart disease: changing shape and shaping change. EMBO Mol Med 7(7): 865-877

29. Schon EA, Przedborski S (2011) Mitochondria: the next (neurode)generation. Neuron 70(6):1033-1053

30. DiMauro S, Schon EA (2003) Mitochondrial respiratory-chain diseases. N Engl J Med 348(26):2656-2668

31. Rossignol R, Faustin B, Rocher C et al (2003) Mitochondrial threshold effects. Biochem J 370(Pt 3):751-762

32. Head B, Griparic L, Amiri M, Gandre-Babbe S, van der Bliek AM (2009) Inducible proteolytic inactivation of OPA1 mediated by the OMA1 protease in mammalian cells. J Cell Biol 187(7):959-966

33. Ehses S, Raschke I, Mancuso G et al (2009) Regulation of OPA1 processing and mitochondrial fusion by m-AAA protease isoenzymes and OMA1. J Cell Biol 187(7):1023-1036
34. Anand R, Wai T, Baker MJ et al (2014) The i-AAA protease YME1L and OMA1 cleave OPA1 to balance mitochondrial fusion and fission. J Cell Biol 204(6):919-929

35. Carelli V, Rugolo M, Sgarbi G et al (2004) Bioenergetics shapes cellular death pathways in Leber's hereditary optic neuropathy: a model of mitochondrial neurodegeneration. Biochim Biophys Acta 1658(1-2):172-179

36. King MP, Attardi G (1989) Human cells lacking mtDNA: repopulation with exogenous mitochondria by complementation. Science 246(4929):500-503

37. Buchet K, Godinot C (1998) Functional F1-ATPase essential in maintaining growth and membrane potential of human mitochondrial DNA-depleted rho degrees cells. J Biol Chem 273(36):22983-22989

38. Patten DA, Wong J, Khacho M et al (2014) OPA1-dependent cristae modulation is essential for cellular adaptation to metabolic demand. EMBO J 33(22):2676-2691

39. Loson OC, Song Z, Chen H, Chan DC (2013) Fis1, Mff, MiD49, and MiD51 mediate Drp1 recruitment in mitochondrial fission. Mol Biol Cell 24(5):659-667

40. Jhun BS, Lee H, Jin ZG, Yoon Y (2013) Glucose stimulation induces dynamic change of mitochondrial morphology to promote insulin secretion in the insulinoma cell line INS-1E. PLoS One 8(4):e60810

41. Gottlieb E, Armour SM, Harris MH, Thompson CB (2003) Mitochondrial membrane potential regulates matrix configuration and cytochrome c release during apoptosis. Cell Death Differ 10(6):709-717

42. Lim ML, Minamikawa T, Nagley P (2001) The protonophore $\mathrm{CCCP}$ induces mitochondrial permeability transition without cytochrome $\mathrm{c}$ release in human osteosarcoma cells. FEBS Lett 503(1):69-74

43. Lemasters JJ, Ramshesh VK (2007) Imaging of mitochondrial polarization and depolarization with cationic fluorophores. Methods Cell Biol 80:283-295

44. Cottet-Rousselle C, Ronot X, Leverve X, Mayol JF (2011) Cytometric assessment of mitochondria using fluorescent probes. Cytometry A 79(6):405-425

45. Nicholls DG (2006) Simultaneous monitoring of ionophore- and inhibitor-mediated plasma and mitochondrial membrane potential changes in cultured neurons. J Biol Chem 281(21):14864-14874

46. Gerencser AA, Chinopoulos C, Birket MJ et al (2012) Quantitative measurement of mitochondrial membrane potential in cultured cells: calcium-induced de- and hyperpolarization of neuronal mitochondria. J Physiol 590(Pt 12):2845-2871

47. Kim N, Ripple MO, Springett R (2012) Measurement of the mitochondrial membrane potential and $\mathrm{pH}$ gradient from the redox poise of the hemes of the bc1 complex. Biophys J 102(5):1194-1203

48. Griparic L, Kanazawa T, van der Bliek AM (2007) Regulation of the mitochondrial dynamin-like protein Opal by proteolytic cleavage. J Cell Biol 178(5):757-764

49. Song Z, Chen H, Fiket M, Alexander C, Chan DC (2007) OPA1 processing controls mitochondrial fusion and is regulated by mRNA splicing, membrane potential, and Yme1L. J Cell Biol 178(5):749-755

50. Guillery O, Malka F, Landes T et al (2008) Metalloproteasemediated OPA1 processing is modulated by the mitochondrial membrane potential. Biol Cell 100(5):315-325

51. MacVicar TD, Lane JD (2014) Impaired OMA1-dependent cleavage of OPA1 and reduced DRP1 fission activity combine to prevent mitophagy in cells that are dependent on oxidative phosphorylation. J Cell Sci 127(Pt 10):2313-2325

52. Quiros PM, Ramsay AJ, Sala D et al (2012) Loss of mitochondrial protease OMA1 alters processing of the GTPase OPA1 and causes obesity and defective thermogenesis in mice. EMBO J 31(9):2117-2133 
53. Cereghetti GM, Costa V, Scorrano L (2010) Inhibition of Drp1dependent mitochondrial fragmentation and apoptosis by a polypeptide antagonist of calcineurin. Cell Death Differ 17(11):1785-1794

54. Cribbs JT, Strack S (2007) Reversible phosphorylation of Drp1 by cyclic AMP-dependent protein kinase and calcineurin regulates mitochondrial fission and cell death. EMBO Rep 8(10):939-944

55. Parone PA, Da Cruz S, Tondera D et al (2008) Preventing mitochondrial fission impairs mitochondrial function and leads to loss of mitochondrial DNA. PLoS One 3(9):e3257

56. Saita S, Ishihara T, Maeda M et al (2016) Distinct types of protease systems are involved in homeostasis regulation of mitochondrial morphology via balanced fusion and fission. Genes Cells 21(5):408-424

57. Mopert K, Hajek P, Frank S et al (2009) Loss of Drp1 function alters OPA1 processing and changes mitochondrial membrane organization. Exp Cell Res 315(13):2165-2180

58. Suen DF, Narendra DP, Tanaka A, Manfredi G, Youle RJ (2010) Parkin overexpression selects against a deleterious mtDNA mutation in heteroplasmic cybrid cells. Proc Natl Acad Sci USA 107(26):11835-11840

59. Huang P, Galloway CA, Yoon Y (2011) Control of mitochondrial morphology through differential interactions of mitochondrial fusion and fission proteins. PLoS One 6(5):e20655

60. Zhang K, Li H, Song Z (2014) Membrane depolarization activates the mitochondrial protease OMA1 by stimulating selfcleavage. EMBO Rep 15(5):576-585

61. Ji WK, Hatch AL, Merrill RA, Strack S, Higgs HN (2015) Actin filaments target the oligomeric maturation of the dynamin GTPase Drp1 to mitochondrial fission sites. Elife 4:e11553

62. Hatch AL, Ji WK, Merrill RA, Strack S, Higgs HN (2016) Actin filaments as dynamic reservoirs for Drp1 recruitment. Mol Biol Cell 27(20):3109-3121

63. Gomes LC, Di Benedetto G, Scorrano L (2011) Essential amino acids and glutamine regulate induction of mitochondrial elongation during autophagy. Cell Cycle 10(16):2635-2639
64. Narendra D, Tanaka A, Suen DF, Youle RJ (2008) Parkin is recruited selectively to impaired mitochondria and promotes their autophagy. J Cell Biol 183(5):795-803

65. Toyama EQ, Herzig S, Courchet J et al (2016) Metabolism. AMP-activated protein kinase mediates mitochondrial fission in response to energy stress. Science 351(6270):275-281

66. Wai T, Garcia-Prieto J, Baker MJ et al (2015) Imbalanced OPA1 processing and mitochondrial fragmentation cause heart failure in mice. Science 350(6265):aad0116

67. Mishra P, Varuzhanyan G, Pham AH, Chan DC (2015) Mitochondrial dynamics is a distinguishing feature of skeletal muscle fiber types and regulates organellar compartmentalization. Cell Metab 22(6): 1033-1044

68. Bender A, Krishnan KJ, Morris CM et al (2006) High levels of mitochondrial DNA deletions in substantia nigra neurons in aging and Parkinson disease. Nat Genet 38(5):515-517

69. Kraytsberg Y, Kudryavtseva E, McKee AC et al (2006) Mitochondrial DNA deletions are abundant and cause functional impairment in aged human substantia nigra neurons. Nat Genet 38(5):518-520

70. Mootha VK, Lindgren CM, Eriksson KF et al (2003) PGC-1alpha-responsive genes involved in oxidative phosphorylation are coordinately downregulated in human diabetes. Nat Genet 34(3):267-273

71. Ritov VB, Menshikova EV, Azuma K et al (2010) Deficiency of electron transport chain in human skeletal muscle mitochondria in type 2 diabetes mellitus and obesity. Am J Physiol Endocrinol Metab 298(1):E49-E58

72. Liu R, Jin P, Liqun Y et al (2014) Impaired mitochondrial dynamics and bioenergetics in diabetic skeletal muscle. PLoS One 9(3):e92810

73. Yamano K, Fogel AI, Wang C, van der Bliek AM, Youle RJ (2014) Mitochondrial Rab GAPs govern autophagosome biogenesis during mitophagy. Elife 3:e01612 